\title{
Origin of Orthorhombic Transition, Magnetic Transition, and Shear Modulus Softening in Iron Pnictide Superconductors: Analysis based on the Orbital Fluctuation Theory
}

\author{
Hiroshi Kontani ${ }^{1}$, Tetsuro SAIto $^{1}$, and Seiichiro OnARI ${ }^{2}$ \\ ${ }^{1}$ Department of Physics, Nagoya University and JST, TRIP, Furo-cho, Nagoya 464-8602, Japan. \\ 2 Department of Applied Physics, Nagoya University and JST, TRIP, Furo-cho, Nagoya 464-8602, Japan.
}

(Dated: May 31, 2018)

\begin{abstract}
The main features in iron-pnictide superconductors are summarized as (i) the orthorhombic transition accompanied by remarkable softening of shear modulus, (ii) high- $T_{\mathrm{c}}$ superconductivity close to the orthorhombic phase, and (iii) stripe-type magnetic order induced by orthorhombicity. To present a unified explanation for them, we analyze the multiorbital Hubbard-Holstein model with Fe-ion optical phonons based on the orbital fluctuation theory. In the random-phase-approximation (RPA), a small electron-phonon coupling constant $(\lambda \sim 0.2)$ is enough to produce large orbital (=charge quadrupole) fluctuations. The most divergent susceptibility is the $O_{x z}$-antiferro-quadrupole (AFQ) susceptibility, which causes the $s$-wave superconductivity without sign reversal ( $s_{++}$-wave state). At the same time, divergent development of $O_{x^{2}-y^{2}}$-ferro-quadrupole (FQ) susceptibility is brought by the "two-orbiton process" with respect to the AFQ fluctuations, which is absent in the RPA. The derived FQ fluctuations cause the softening of $C_{66}$ shear modulus, and its long-range-order not only triggers the orthorhombic structure transition, but also induces the instability of stripetype antiferro-magnetic state. In other words, the condensation of composite bosons made of two orbitons gives rise to the FQ order and structure transition. The theoretically predicted multiorbital-criticality presents a unified explanation for abovementioned features of iron pnictide superconductors.

PACS numbers: 74.70.Xa, 74.20.-z, 74.20.Rp, 74.25.Kc
\end{abstract}

\section{INTRODUCTION}

In iron pnictide superconductors ${ }^{1}$, both spin and orbital degrees of freedom play important roles on various electronic properties, such as the high- $T_{\mathrm{c}}$ superconductivity, orthorhombic structure transition, and magnetic transition. As for the origin of the superconductivity, fully-gapped sign-reversing $s$-wave ( $s_{+}$-wave) state had been studied based on spin fluctuation theories ${ }^{2-5}$. The origin of the spin fluctuations is the intra-orbital nesting and the Coulomb interaction. However, the robustness of $T_{\mathrm{c}}$ against randomness in iron pnictides indicates the absence of sign-reversal in the superconducting (SC) gap $^{6-8}$.

Later, orbital-fluctuation-mediated $s$-wave state without sign reversal ( $s_{++}$-wave) had been proposed based on the Hubbard-Holstein $(\mathrm{HH})$ model $^{9-11}$. The origin of the orbital fluctuations is the inter-orbital nesting and the electron-phonon ( $e$-ph) interactions due to non- $A_{1 g}$ optical phonons. One of the merits of this scenario is the robustness of the $s_{++}$-wave state against impurities. Another merit is that the close relation between $T_{\mathrm{c}}$ and the crystal structure revealed by Lee ${ }^{12}, e . g ., T_{\mathrm{c}}$ becomes the highest when the $\mathrm{As}_{4}$ cluster is regular tetrahedron, is automatically explained ${ }^{10}$. Moreover, orbital-fluctuationmediated $s_{++}$-wave state scenario is consistent with the large SC gap on the $z^{2}$-orbital band in Ba122 systems ${ }^{10}$, observed by bulk-sensitive laser ARPES measurement ${ }^{13}$.

The "resonance-like" hump structure in the neutron inelastic scattering ${ }^{14}$ is frequently explained as the spinresonance due to the sign reversal in the $\mathrm{SC} \operatorname{gap}^{15,16}$.
However, experimental hump structure is well reproduced in terms of the $s_{++}$-wave SC state, rather than the $s_{ \pm}$-wave $\mathrm{SC}$ state, by taking the suppression in the inelastic scattering $\gamma(\omega)$ for $|\omega| \leq 3 \Delta$ in the SC state

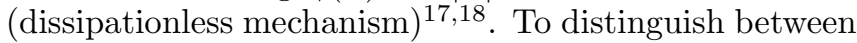
both SC states, measurements of phonon spectral function for $|\omega| \lesssim 2 \Delta$ would be useful ${ }^{19}$. In the normal state, prominent non-Fermi liquid transport phenomena in $\rho$ and $R_{\mathrm{H}}{ }^{20}$ are frequently ascribed to the evidence of spin fluctuations ${ }^{21}$. However, they are also explained by the development of antiferro-orbital fluctuations ${ }^{11}$.

In order to identify the mechanism of superconductivity, we have to understand the origin of the ordered state, although it is still unsolved in iron pnictides. For example, in many heavy fermion superconductors, the SC phase appears next to the spin-densitywave (SDW) state, indicating the occurrence of spinfluctuation-mediated superconductivity. In contrast, the ordered state in iron pnictides is not a simple SDW state: In fact, the tetragonal to orthorhombic structure transition occurs at $T_{S} \sim 100 \mathrm{~K}$, usually above the SDW transition temperature $T_{\mathrm{N}}{ }^{22}$. In addition, large imbalance of $x z$ - and $y z$-orbitals at the Fermi level and reconstruction of the Fermi surfaces (FSs) had been observed by angle resolved photoemission spectroscopy (ARPES) measurements ${ }^{23-25}$. These results indicate that the ferro orbital-density-wave (ODW) is the origin of the orthorhombic structure transition. The SDW state may originate from the in-plane anisotropy in the exchange interaction $\left(J_{1 a} \neq J_{1 b}\right)$ associated with the ODW order ${ }^{26}$.

In addition, prominent symmetry breaking $C_{4} \rightarrow C_{2}$ 
is realized in detwinned 122 systems even above $T_{S}$ and $T_{\mathrm{N}}$, under very small uniaxial pressure. For example, it is recognized as the large in-plane anisotropy in the resistivity ${ }^{27,28}$ and the optical conductivity ${ }^{29}$ at $T^{*} \sim$ $200 \mathrm{~K}$, which is much higher than $T_{S}$ and $T_{\mathrm{N}}$. Moreover, the reconstruction of the FSs starts at $T^{*}$ in detwinned $\mathrm{Ba}\left(\mathrm{Fe}_{1-x} \mathrm{Co}_{x}\right)_{2} \mathrm{As}_{2}{ }^{25}$. The discovery of these "electronic nematic phase" would indicate that the the ODW fluctuations deveolop divergently above $T_{S}$, and the structure transition is (almost) the second-order.

Recently, prominent softening of shear modulus in undoped and under-doped $\mathrm{Ba}\left(\mathrm{Fe}_{1-x} \mathrm{Co}_{x}\right)_{2} \mathrm{As}_{2}$ had been reported by acoustic measurements ${ }^{30,31}$. Yoshizawa et $a l^{31}$ observed all shear moduli $C_{44}, C_{66}$ and $C_{E}$. Recently, they had also performed systematic measurement for $x=0 \sim 0.225$, and found that only $C_{66}$ shows the prominent softening in both under- and over-doped systems $^{32}$. Similar results were reported by Goto et al. independently ${ }^{33}$. This observation can be explained by the development of ferro-orbital fluctuations ${ }^{10}$ or spinnematic fluctuations ${ }^{30}$. Considering large quadrupolestrain coupling in iron pnictides, all the observations mentioned above suggest the importance of orbital physics, and pose a serious challenge for theories of iron pnictide superconductors. In fact, Goto et al. have shown that $C_{66}$ is almost independent of the magnetic field up to $\sim 50 \mathrm{~T}$, indicating the non-magnetic origin of the softening ${ }^{33}$.

Thus, the main features of the iron-pnictide superconductors would be summarized as (i) the orthorhombic (or nematic) transition accompanied by remarkable $C_{66}$ softening, (ii) emergence of high- $T_{\mathrm{c}}$ superconductivity next to the orthorhombic phase, and (iii) the stripe-type magnetic order induced by the orthorhombicity. The unified explanation has not been achieved as far as we know.

In this paper, we develop the orbital fluctuation theory to explain abovementioned features (i)-(iii) based on the random-phase-approximation (RPA) and beyond RPA. In the RPA, large $O_{x z}$-antiferro-quadrupole (AFQ) fluctuations are produced by the $e$-ph interactions, while they do not produce the softening of $C_{66}$ nor $C_{44}$. If we go beyond the RPA, however, we find that the $O_{x^{2}-y^{2}}$ ferro-quadrupole (FQ) fluctuations are brought by the "two-orbiton process" near the AFQ quantum-criticalpoint (QCP). The induced FQ fluctuations cause the softening of $C_{66}$, and the commensurate ferro-orbital order is realized at $T=T_{S}$. It is predicted that the AFQ-QCP is located at the FQ-QCP, which is the endpoint of the orthorhombic phase. Near this multi-orbital QCPs, the superconductivity is mainly caused by the AFQ fluctuations, and the orthorhombic transition is brought by the FQ fluctuations. Moreover, the stripetype antiferro-magnetic state is induced in the orbitalordered state, since the orbital polarization gives strong in-plane anisotropy in the spin-nesting. The present study gives a microscopic justification for the anisotropic Heisenberg model description in the SDW state ${ }^{26,34}$.

There is a long history in the study of supercon- ductivity due to charge or orbital fluctuations in multiorbital systems, starting from the exciton-assisted superconductivity $^{35-39}$. In multiorbital systems, on-site Coulomb interaction is composed of the intra-orbital term $U$, inter-orbital one $U^{\prime}$, and Hund's or exchange term $J$. Since $U$ is usually larger than $U^{\prime} \approx U-2 J$, spin fluctuations induced by $U$ give non $s$-wave $\mathrm{SC}$ states. However, Takimoto et $a l^{40}$ had shown that charge (or orbital) fluctuations induced by $U^{\prime}$ give conventional $s$ wave SC state if the relations $U^{\prime}>U$ and $J \sim 0$ are assumed. Although this idea was applied to pnictides ${ }^{41}$, the relation $U^{\prime} \sim 0.6 U$ is realized in many compounds ${ }^{42}$. Even if $U^{\prime} \sim 0.6 U$ and $J \sim 0.2 U$, the present authors had shown that the orbital-fluctuation-mediated superconductivity is realized by quadrupole-quadrupole interaction mediated by non- $A_{1 g}$ phonons, which works as the "negative effective exchange $J_{\text {eff }}$ for the charge sector." 9 . In iron pnictides, orbital fluctuations develop even when the dimensionless $e$-ph coupling $\lambda$ due to Fe-ion optical phonons is just $\sim 0.2$, according to the RPA ${ }^{10}$ and FLEX approximation ${ }^{11}$. As for As-ion $A_{1 g}$ mode ${ }^{41}$, orbital fluctuations develop only when $\lambda_{A_{1 g}} \sim 1$, which is unrealistic in iron pnictides.

Recently, Yanagi et al. ${ }^{43}$ added the "orthorhombic phonon" to the HH model proposed by the present authors $^{9-11}$, and studied the ferro-orbital fluctuations. However, neither high- $T_{\mathrm{c}}$ nor structure transition are explained by their theory: First, the "orthorhombicphonon" is acoustic $\left(\omega_{\boldsymbol{q}} \propto|\boldsymbol{q}|\right)$ although it is treated as optical in Ref. 43 incorrectly. Their theory belongs to the cooperative Jahn-Teller structure transition due to acoustic phonons like manganites; see details in Appendix A. In this case, the energy-scale of "ferro-orbital fluctuations" is too low $\left(\sim \omega_{q}\right)$ to explain high- $T_{\mathrm{c}}$, since experimental $T_{\mathrm{c}}$ is much higher than $\omega_{\boldsymbol{q}} \sim 10 \mathrm{~K}$ for $|\boldsymbol{q}| \sim$ $0.1 \pi^{44,45}$. Small orthorhombicity $(a-b) /(a+b) \sim 0.003$ in iron pnictides is also inconsistent with the cooperative Jahn-Teller scenerio. Second, the derived orbital order is "incommensurate" 46 , which is inconsistent with the orthorhombic structure transition and the $C_{66}$ softening.

These problems are resolved in the present theory since both high-energy AFQ and low-energy "commensurate" FQ fluctuations develop at the same time, without the necessity of fine-tuning model parameters. The former (latter) fluctuations give the superconductivity (orthorhombic transition).

\section{MODEL HAMILTONIAN}

First, we shortly explain the relation between fiveorbital and ten-orbital models for iron pnictides. In this study, we set $x$ and $y$ axes parallel to the nearest Fe-Fe bonds, and represent the $z^{2}, x z, y z, x y$, and $x^{2}-y^{2}$ $d$-orbitals in the $x y z$-coordinate as $1,2,3,4$, and 5 , respectively. The FSs are mainly composed of $t_{2 g}$ orbitals $(l=2 \sim 4)$, although $e_{g}$ orbitals also play non-negligible roles in producing orbital fluctuations ${ }^{10}$. Figure 1 shows 
the crystal structure of FeAs-plane. Since the As-A (As$\mathrm{B})$ ions form the upper- (lower-) plane, the unit cell contains Fe-A and Fe-B, which we call the two-iron unit cell. Since each Fe-ion contains five-orbitals, the original tight-binding model for iron-pnictides is given as the "ten-orbital model".

In Refs. 2,42, the authors introduced the gauge transformation $|2,3\rangle \rightarrow-|2,3\rangle$ only for Fe-B sites, which we call the "unfold-gauge transformation". Due to this gauge transformation, the unit cell of the kinetic term is halved to become the single-iron unit cell, shown in Figure 1. In the obtained "five-orbital model", the kinetic term is given as

$$
H_{0}=\sum_{i j ; l m ; \sigma} t_{l m}^{i j} c_{i, l \sigma}^{\dagger} c_{j, m \sigma}
$$

where $i, j$ denotes the unit cell, $l, m=1 \sim 5$ represent the $d$-orbital, and $\sigma= \pm 1$ is the spin index. $c_{i, l \sigma}^{\dagger}$ is the creation operator of the $d$-electron, and $t_{l m}^{i j}$ with $i \neq j$ $(i=j)$ is the hopping integral (local potential). This fiveorbital model is convenient to study the Eliashberg gap equation $^{2,9}$. In studying the orbital physics, however, we have to keep the fact in mind that the sign of quadrupole operators $\hat{O}_{x z}$ and $\hat{O}_{y z}$ at Fe-B sites are reversed by the unfold-gauge transformation. By taking care of this fact, we study the softening of shear moduli based on the fiveorbital model hereafter. In Appendix B, we calculate the orbital fluctuations using the original ten-orbital model, and make comparison between results of two models.

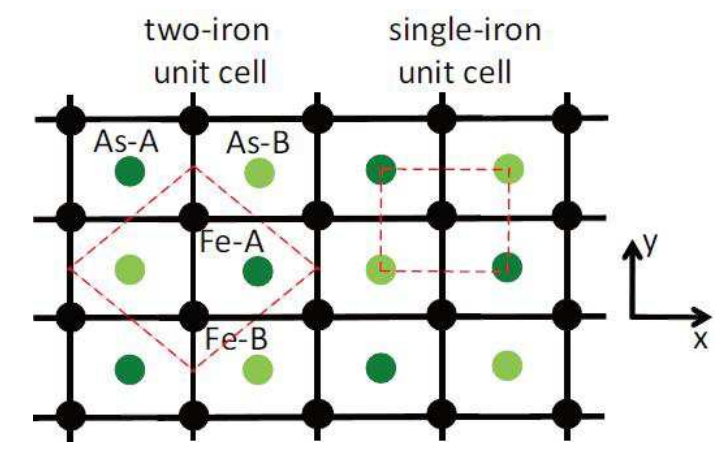

FIG. 1: (Color online) Crystal structure of FeAs-layer, in which the unit cell is given by the two-iron unit cell composed of Fe-A, Fe-B, As-A, and As-B. The single-iron unit cell is realized by applying the "unfold-gauge transformation".

Figure 2 shows the FSs in the (a) five-orbital model and the (b) ten-orbital model. The FSs in (a) coincide with the FSs in (b) if we fold the former FSs into the twoiron Brillouin zone (BZ). We use the hopping parameters for LaOFeAs given in Ref. 2. The colors correspond to 2 (green), 3 (red), and 4 (blue), respectively. The interorbital nesting between the orbital 2 on FS $\alpha_{2}$ and orbital 4 on FS $\beta_{2}$ causes the most divergent AFQ fluctuations.

Next, we introduce the $e$-ph interaction due to Fe-ion Einstein optical modes. The Hamiltonian given in Eq.
(4) of Refs. 10 is simply rewritten as the following bilinear form in the $x y z$-coordinate:

$$
H_{e-\mathrm{ph}}=\eta \sum_{i}\left(\hat{O}_{y z}^{i} u_{x}^{i}+\hat{O}_{x z}^{i} u_{y}^{i}+\hat{O}_{x y}^{i} u_{z}^{i}\right)
$$

where $\eta=60 e^{2} a_{d}^{2} / 7 \sqrt{3} R_{\mathrm{Fe}-\mathrm{As}}^{4} ; a_{d}$ is the radius of $d$ orbital (e.g., Shannon crystal radius of $\mathrm{Fe}^{2+}$ is $0.77 \AA$ ), and $R_{\mathrm{Fe}-\mathrm{As}} \approx 2.4 \AA$. $\boldsymbol{u}^{i}$ is the displacement vector of the $i$-th Fe-ion, and $\hat{O}_{\Gamma}^{i}(\Gamma=x z, y z, x y)$ is the charge quadrupole operator given as

$$
\hat{O}_{\Gamma}^{i} \equiv \sum_{l m}^{ \pm} o_{\Gamma}^{l, m} \hat{m}_{l, m}^{i}
$$

where $\hat{m}_{l, m}^{i} \equiv \sum_{\sigma} c_{i, l \sigma}^{\dagger} c_{i, m \sigma}$, and the coefficient is defined as $o_{x z}^{l, m}=7\langle l|\hat{x} \hat{z}| m\rangle$ for $\Gamma=x z$, where $\hat{\boldsymbol{x}}=x / r$ and so on. The non-zero coefficients are given as

$$
\begin{aligned}
& o_{x z}^{2,5}=o_{x z}^{3,4}=\sqrt{3} o_{x z}^{1,2}=1 \\
& -o_{y z}^{3,5}=o_{y z}^{2,4}=\sqrt{3} o_{y z}^{1,3}=1 \\
& o_{x y}^{2,3}=-\sqrt{3} o_{x y}^{1,4} / 2=1 .
\end{aligned}
$$

Be careful not to confuse $\hat{O}_{x z}$ with the $x z$-orbital operator. Other two quadrupole operators are $O_{z^{2}}$ and $O_{x^{2}-y^{2}}$, whose coefficients are respectively defined as $o_{x^{2}-y^{2}}^{l, m}=$ $(7 / 2)\left\langle l\left|\left(\hat{x}^{2}-\hat{y}^{2}\right)\right| m\right\rangle$ and $o_{z^{2}}^{l, m}=(7 / 2 \sqrt{3})\left\langle l\left|\left(3 \hat{z}^{2}-1\right)\right| m\right\rangle$. (They are written as $O_{2}^{0}$ and $O_{2}^{2}$ in literatures.) The non-zero coefficients are given as

$$
\begin{aligned}
& o_{x^{2}-y^{2}}^{2,2}=-o_{x^{2}-y^{2}}^{3,3}=-(\sqrt{3} / 2) o_{x^{2}-y^{2}}^{1,5}=1 \\
& o_{z^{2}}^{1,1}=2 o_{z^{2}}^{2,2}=2 o_{z^{2}}^{3,3}=-o_{z^{2}}^{4,4}=-o_{z^{2}}^{5,5}=2 / \sqrt{3}
\end{aligned}
$$

Expect for $\Gamma=z^{2}$, all the matrix elements of $\hat{o}_{\Gamma}$ with respect to the $t_{2 g}$-orbital $(2 \sim 4)$ are \pm 1 .

Here, we derived the $e$-ph interaction based on the point-charge model. Although $e$-ph interaction is also induced by the change in the $d-p$ hopping, as discussed in Ref. 47, we expect it is small since the weight of $p$-electron on the Fermi surface is just $\sim 5 \%$ in iron pnictides. Fortunately, because of the Wigner-Eckart theorem, the matrix elements of the local quadrupole-phonon interaction is always given by the quadrupole operator $\hat{O}_{\Gamma}^{i}$, independently of the details of the interaction. Since the magnitude of the hexadecapole-phonon interaction is $\left(a_{d} / R_{\mathrm{Fe}-\mathrm{Fe}}\right)^{2} \sim 0.1$ times that of the quadrupole-phonon interaction, we can safely use Eq. (2).

Equation (2) means that the displacement $u_{x}$ produces the quadrupole potential $\hat{O}_{y z}$, which causes the scattering of electrons between orbitals 2 and 4 . The $e$-ph interactions in Eq. (2) within $t_{2 g}$-orbitals are shown in Fig. 3. Then, the phonon-mediated el-el interaction $V_{\mathrm{el}-\mathrm{el}}^{\mathrm{ph}}$ is obtained by taking the contraction of $\boldsymbol{u}^{i}$, which gives the local phonon Green function $D(\tau) \equiv\left\langle T_{\tau} u_{\mu}^{i}(\tau) u_{\mu}^{i}(0)\right\rangle$ 
(a)

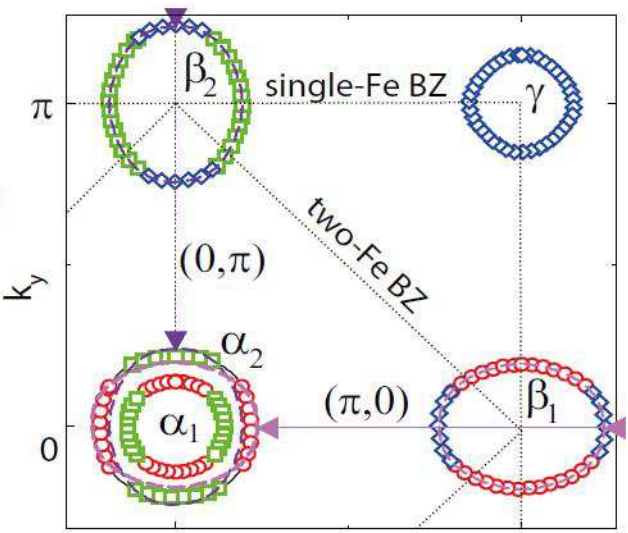

(b)

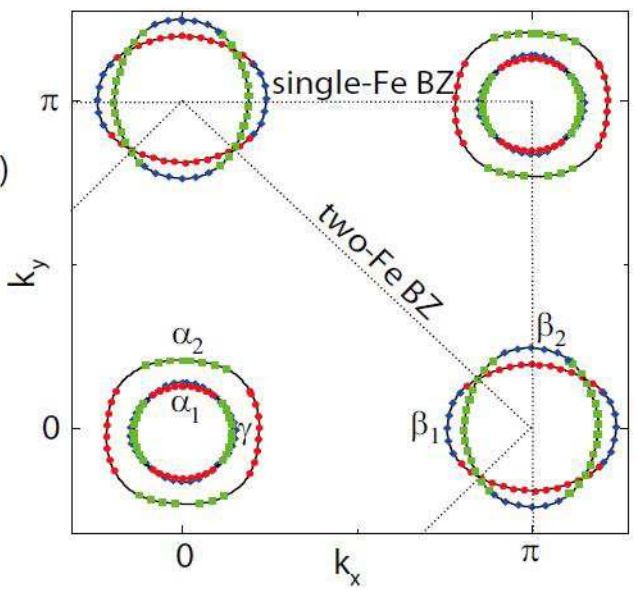

FIG. 2: (Color online) (a) FSs for $n=6.0$ in the unfolded model. The colors correspond to 2 (green), 3 (red), and 4 (blue), respectively. The inter-orbital nesting between FS $\alpha_{2}$ (green) and FS $\beta_{2}$ (blue) causes the AFQ fluctuations. (b) FSs for the original ten-orbital model.

$(\mu=x, y, z)$. By taking the Fourier transformation, we obtain

$$
D\left(\omega_{l}\right)=\frac{2\left\langle u^{2}\right\rangle_{0} \omega_{D}}{\omega_{l}^{2}+\omega_{D}^{2}}
$$

where $\omega_{l}=2 \pi T l$ is the Boson Matsubara frequency, $\omega_{D}$ is the optical phonon frequency, and $\sqrt{\left\langle u^{2}\right\rangle_{0}}=$ $\sqrt{1 / 2 M_{\mathrm{Fe}} \omega_{D}}$ is the uncertainty in position for Fe ions; $\sqrt{\left\langle u^{2}\right\rangle_{0}}=0.044 \AA$ for $\omega_{\mathrm{D}}=0.02 \mathrm{eV}^{10}$. Then, $V_{\mathrm{el}-\mathrm{el}}^{\mathrm{ph}}$ is expressed as the following quadrupole-quadrupole interaction:

$V_{\mathrm{el}-\mathrm{el}}^{\mathrm{ph}}=-g\left(\omega_{l}\right) \sum_{i}\left\{\hat{O}_{y z}^{i} \cdot \hat{O}_{y z}^{i}+\hat{O}_{x z}^{i} \cdot \hat{O}_{x z}^{i}+\hat{O}_{x y}^{i} \cdot \hat{O}_{x y}^{i}\right\}$

where $g\left(\omega_{l}\right)=g \cdot \omega_{\mathrm{D}}^{2} /\left(\omega_{l}^{2}+\omega_{\mathrm{D}}^{2}\right)$, and $g$ is the phononmediated el-el interaction at zero frequency; $g=0.34 \mathrm{eV}$ in the present point-charge model ${ }^{9,10}$. (a)

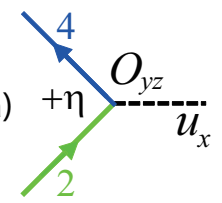

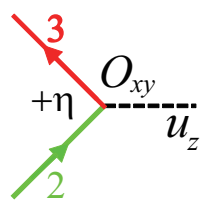

FIG. 3: (Color online) Inter-orbital scattering processes due to the $e$-ph interaction by $u_{\mu}(\mu=x, y, z)$ within the $t_{2 g^{-}}$ orbitals $(l=2 \sim 4)$.

\section{RANDOM-PHASE-APPROXIMATION}

Now, we explain the RPA for the five-orbital $\mathrm{HH}$ model $^{40}$. The irreducible susceptibility in the five orbital model is given by

$$
\chi_{l l^{\prime}, m m^{\prime}}^{0}(q)=-\frac{T}{N} \sum_{k} G_{l m}^{0}(k+q) G_{m^{\prime} l^{\prime}}^{0}(k)
$$

where $\hat{G}^{0}(k)=\left[i \epsilon_{n}+\mu-\hat{H}_{k}^{0}\right]^{-1}$ is the $d$ electron Green function in the orbital basis, $q=\left(\boldsymbol{q}, \omega_{l}\right), k=\left(\boldsymbol{k}, \epsilon_{n}\right)$, and $\epsilon_{n}=(2 n+1) \pi T$ is the fermion Matsubara frequency. $\mu$ is the chemical potential, and $\hat{H}_{\boldsymbol{k}}^{0}$ is the kinetic term. Then, the susceptibilities for spin and charge sectors in the RPA are given as $^{40}$

$$
\begin{gathered}
\hat{\chi}^{s}(q)=\frac{\hat{\chi}^{0}(q)}{1-\hat{\Gamma}^{s} \hat{\chi}^{0}(q)}, \\
\hat{\chi}^{c}(q)=\frac{\hat{\chi}^{0}(q)}{1-\hat{\Gamma}^{c}\left(\omega_{l}\right) \hat{\chi}^{0}(q)},
\end{gathered}
$$

where the bare four-point vertices $\hat{\Gamma}^{s, c}$ are

$$
\begin{gathered}
\Gamma_{l_{1} l_{2}, l_{3} l_{4}}^{s}= \begin{cases}U, & l_{1}=l_{2}=l_{3}=l_{4} \\
U^{\prime}, & l_{1}=l_{3} \neq l_{2}=l_{4} \\
J, & l_{1}=l_{2} \neq l_{3}=l_{4} \\
J^{\prime}, & l_{1}=l_{4} \neq l_{2}=l_{3}\end{cases} \\
\hat{\Gamma}^{c}\left(\omega_{l}\right)=-\hat{C}-2 \hat{V}_{\mathrm{el}-\mathrm{el}}^{\mathrm{ph}}\left(\omega_{l}\right), \\
C_{l_{1} l_{2}, l_{3} l_{4}}= \begin{cases}U, & l_{1}=l_{2}=l_{3}=l_{4} \\
-U^{\prime}+2 J, & l_{1}=l_{3} \neq l_{2}=l_{4} \\
2 U^{\prime}-J, & l_{1}=l_{2} \neq l_{3}=l_{4} \\
J^{\prime}, & l_{1}=l_{4} \neq l_{2}=l_{3}\end{cases}
\end{gathered}
$$

In Eq. $\quad(15), \quad\left(V_{\mathrm{el}-\mathrm{el}}^{\mathrm{ph}}\right)_{l_{1}, l_{2}, l_{3}, l_{4}}=$ $-g\left(\omega_{l}\right) \sum_{\Gamma}^{x z, y z, y x} o_{\Gamma}^{l_{1}, l_{2}} o_{\Gamma}^{l_{3}, l_{4}}$. Here, we neglect the ladder-diagram for phonon-mediated interaction because of the relation $\omega_{D} \ll W_{\text {band }}{ }^{9}$.

In the RPA, the enhancement of the spin susceptibility $\hat{\chi}^{s}$ is mainly caused by the intra-orbital Coulomb interaction $U$, using the "intra-orbital nesting". On the other hand, the enhancement of $\hat{\chi}^{c}$ in the present model 
is caused by the phonon-induced quadrupole-quadrupole interaction in Eq. (10), utilizing the "inter-orbital nesting" in the present model. The SDW (ODW) state is realized when the spin (charge) Stoner factor $\alpha_{s(c)}$, which is the maximum eigenvalue of $\hat{\Gamma}^{s(c)} \hat{\chi}^{0}(\boldsymbol{q}, 0)$, is unity. When $n=6.05$, the critical value of $U$ is $U_{c}=1.26 \mathrm{eV}$, and the critical value of $g$ (at $U=0$ ) is $g_{c}=0.233 \mathrm{eV}$. Smallness of $g_{\mathrm{c}}$ in iron pnictides originates from the better interorbital nesting. Hereafter, we set the unit of energy as $\mathrm{eV}$ unless otherwise noted.

Here, we introduce the diagonal charge quadrupole susceptibilities in the five-orbital model as

$$
\chi_{\Gamma}^{Q}(q)=\sum_{l l^{\prime}} \sum_{m m^{\prime}} o_{\Gamma}^{l l^{\prime}} \chi_{l l^{\prime} m m^{\prime}}^{c}(q) o_{\Gamma}^{m m^{\prime}}
$$

for $\Gamma=x z, y z, x y$. Their momentum dependence at zero frequency is shown in Fig. 4 for $\alpha_{c}=0.98$. More generally, the quadrupole susceptibility is defined as

$$
\chi_{\Gamma, \Gamma^{\prime}}^{Q}(q)=\sum_{l l^{\prime}} \sum_{m m^{\prime}} o_{\Gamma}^{l l^{\prime}} \chi_{l l^{\prime} m m^{\prime}}^{c}(q) o_{\Gamma^{\prime}}^{m m^{\prime}}
$$

However, its off-diagonal terms with $\Gamma \neq \Gamma^{\prime}$ are negligibly small in the present model in the "xyz-coordinate". In this approximation, in the absence of Coulomb interaction, the quadrupole susceptibility in the RPA is given as

$$
\chi_{\Gamma}^{Q}(q) \approx \chi_{\Gamma}^{Q, 0}(q) /\left(1-2 g \chi_{\Gamma}^{Q, 0}(q)\right),
$$

where $\chi_{\Gamma}^{Q, 0}$ is the irreducible quadrupole susceptibility. Considering the fact that $\chi_{l l^{\prime}, m m^{\prime}}^{0}(q)$ takes large value for $l=m$ and $l^{\prime}=m^{\prime}$, we obtain $\chi_{x z}^{Q, 0}(q) \approx 2 \chi_{25,25}^{0}(q)+$ $2 \chi_{34,34}^{0}(q)+(2 / 3) \chi_{12,12}^{0}(q)$. Since $\chi_{x z}^{Q, 0}(q) \approx 2.5$ at $\boldsymbol{q}=$ $(\pi, 0)$, the critical value of $g$ is $g_{c} \sim 0.2$ in the present model. We stress that the relations $\chi_{\Gamma, \Gamma^{\prime}}^{Q}(q)=\chi_{\Gamma}^{Q}(q) \delta_{\Gamma, \Gamma^{\prime}}$ and Eq. (19) holds exactly for $q=\left(\mathbf{0}, \omega_{l}\right)$. We utilize this relation in calculating the shear modulus.

As for the contributions by $t_{2 g}$-orbitals $(l=2 \sim 4)$, $\chi_{x z}^{Q}(q) \propto \chi_{34,34}^{c}(q), \chi_{y z}^{Q}(q) \propto \chi_{24,24}^{c}(q)$, and $\chi_{x y}^{Q}(q) \propto$ $\chi_{23,23}^{c}(q)$. In Fig. $4(\mathrm{a}), \chi_{x z}^{Q}(\boldsymbol{q})$ has the highest peak at $\boldsymbol{q}=(\pi, 0)$, which is given by the inter-orbital nesting between orbital 3 on FS $\alpha_{2}$ and orbital 4 on FS $\beta_{1}$ in the five-orbital model in Fig. 2 (a). Also, $\chi_{y z}^{Q}(\boldsymbol{q})$ has the highest peak at $\boldsymbol{q}=(0, \pi)$ in Fig. $4(\mathrm{~b})$, due to the inter-orbital nesting between orbital 2 on FS $\alpha_{2}$ and the orbital 4 on FS $\beta_{2}$. We will see that $\chi_{x z(y z)}^{Q}(\boldsymbol{q})$ is modified by the unfolding procedure.

Also, $\chi_{x y}^{Q}(\boldsymbol{q})$ in Fig. 4 (c) is given by the inter-orbital nesting between orbital 2 and 3, due to the out-of-plane oscillations of Fe-ions. The inter-band and intra-band scattering processes produce the enhancement of $\chi_{x y}^{Q}(\boldsymbol{q})$ at $\boldsymbol{q}=(\pi, 0),(0, \pi)$ and $\boldsymbol{q}=\mathbf{0}$, respectively. We note that $\chi_{x y}^{Q}(\boldsymbol{q})$ is not affected by the unfolding procedure.

Figure 5 shows both $\chi_{x z}^{Q}(\boldsymbol{q})$ at $\boldsymbol{q}=(\pi, 0)$ and $\chi_{x y}^{Q}(\mathbf{0})$ as a function of $\alpha_{c}$ for $n=6.0$ and $n=6.05$ given by the
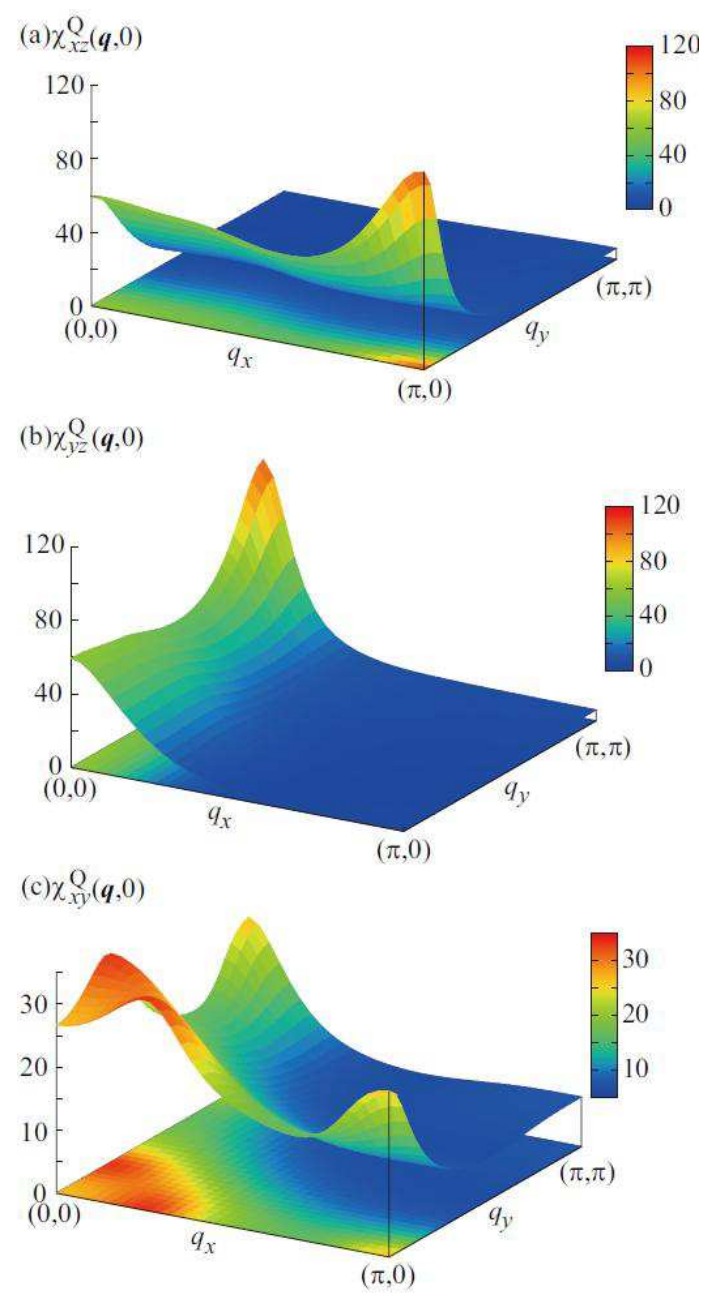

FIG. 4: (Color online) Quadrupole susceptibilities in the fiveorbital model for (a) $\chi_{x z}^{Q}(\boldsymbol{q})$, (b) $\chi_{y z}^{Q}(\boldsymbol{q})$, and (c) $\chi_{x y}^{Q}(\boldsymbol{q})$, respectively. The used model parameters are $n=6.05$, $T=0.05$, and $g=0.22\left(\alpha_{c}=0.98\right)$. The correlation length in (a) or (b) is derived as $\xi=\pi / \Delta q \sim 3$, where $\Delta q$ is the half-width of the peak. Therefore, we obtain the relations $6 \xi^{2} \sim\left(1-\alpha_{c}\right)^{-1}$ and $c \xi^{2} \sim 2\left(1-\alpha_{c}\right)^{-1} \sim 12 \xi^{2}$.

RPA. We see that $\chi_{x z}^{Q}(\boldsymbol{q})$ develops divergently in proportion to $\left(1-\alpha_{c}\right)^{-1} \propto\left(g_{c}-g\right)^{-1}$, while $\chi_{x y}^{Q}(\mathbf{0})$ shows an enhanced but saturated value even at $g=g_{\mathrm{c}}$.

In Appendix B, we will calculate $\chi_{\Gamma}^{Q}(q)$ in the tenorbital model, and make comparison to Fig. 4 in the five-orbital model: Although both results coincide for $\Gamma=x y, z^{2}, x^{2}-y^{2}$, they are different for $\Gamma=x z, y z$. The reason is that the signs of $O_{x z / y z}$ at Fe-B sites are changed by applying the "unfold-gauge transformation". As we will explain in Appendix B, the development of $\chi_{x z / y z}^{Q}(\mathbf{0}, 0)$ in Fig. 4 is the artifact of the unfold-gauge transformation. For this reason, the correct AFQ susceptibility in the ten-orbital model is given as $\chi_{x z / y z}^{Q}(\boldsymbol{q})=\chi_{x z / y z}^{Q, 5-\text { orbital }}(\boldsymbol{q}+(\pi, \pi))$. The optical modes that give the enhancements of $\chi_{y z}^{Q}(\boldsymbol{q})$ at $\boldsymbol{q}=(\pi, 0)$ and $(\pi, \pi)$ in the ten-orbital model $(\boldsymbol{q}=(0, \pi)$ and $(0,0)$ in 


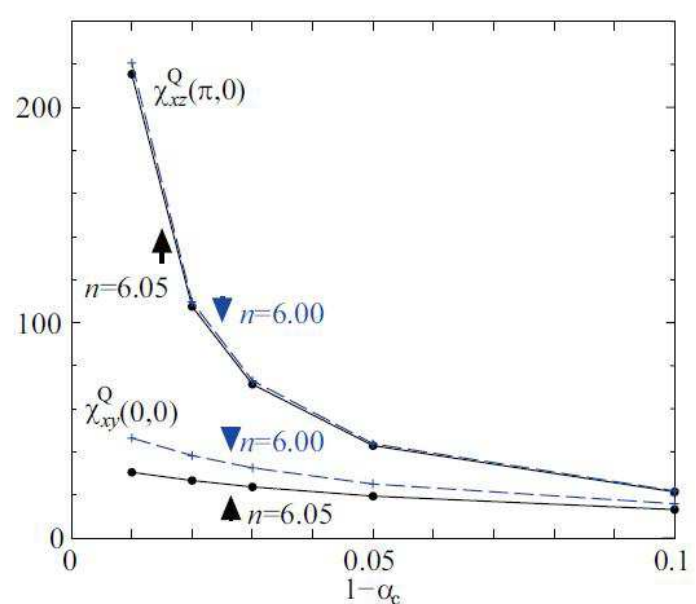

FIG. 5: (Color online) Quadrupole susceptibilities as a function of $\alpha_{c}$ for $n=6.0$ and $n=6.05$ given by RPA. Model parameters are $U=0.8$ and $T=0.05$. We can recognize the relation $\chi_{x z}^{Q}(\boldsymbol{Q}) \propto\left(1-\alpha_{c}\right)^{-1} \propto\left(g_{\mathrm{c}}-g\right)^{-1}$, which diverges at $\alpha_{c}=1$ or $g=g_{\mathrm{c}}$.

the five-orbital model) are caused by the in-plane $u_{x}$ oscillations shown in Fig.6 (a). Also, the enhancements of $\chi_{x y}^{Q}(\boldsymbol{q})$ at $\boldsymbol{q}=(\pi, 0)$ and $(0,0)$ are caused by the out-ofplane $u_{z}$ oscillations in Fig.6 (b).

(a)

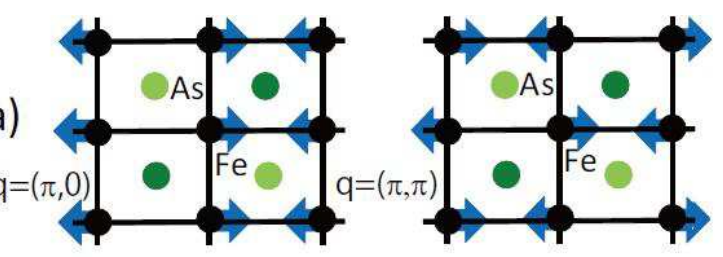

(b)

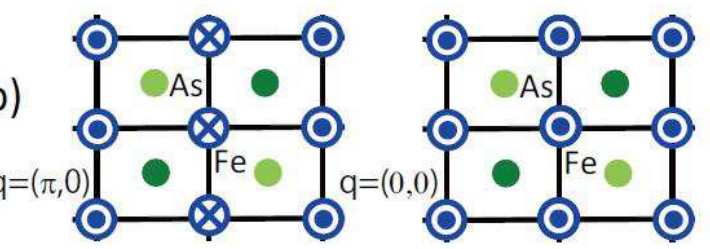

FIG. 6: (Color online) (a) Fe-ion in-plane optical phonons with momentum $\boldsymbol{q}=(\pi, 0)$ and $\boldsymbol{q}=(\pi, \pi)$. (b) Fe-ion outof-plane optical phonons with momentum $\boldsymbol{q}=(\pi, 0)$ and $\boldsymbol{q}=$ $(0,0)$.

\section{ACOUSTIC PHONONS}

In previous sections, we studied the $e$-ph interaction due to optical phonons, and calculated the quadrupole susceptibilities by the RPA. To obtain the shear modulus, we also need the knowledge on the $e$-ph interaction due to acoustic phonons with momentum $\boldsymbol{q} \approx 0$. In fact, shear modulus is proportional to the square of the acoustic phonon velocity, which is renormalized by the electron-acoustic phonon interaction in the presence of strong quadrupole fluctuations. In this section, we derive the $e$-ph interaction due to acoustic phonons with $\boldsymbol{q} \approx 0$. Hereafter, we use the unit $\hbar=1$, and take the nearestneighbor $\mathrm{Fe}-\mathrm{Fe}$ distance $a_{\mathrm{Fe}-\mathrm{Fe}}$ as the unit of length.

Figure 7 shows the transverse acoustic modes that are related to (a) $C_{44}$, (b) $C_{E}=\left(C_{11}-C_{12}\right) / 2$, and (c) $C_{66}{ }^{31}$. Now, we calculate the $e$-ph interaction based on the point-charge model, by following the procedure in Ref. 9 for the optical phonons. The quadrupole potential energies at Fe-site caused by the transverse acoustic phonons in Fig. 7 are given as

$$
\begin{aligned}
V_{44} & =-\frac{3 e^{2}}{R_{\mathrm{Fe}-\mathrm{As}}^{4}} \frac{8}{\sqrt{3}} x z \cdot \tilde{u}_{44}, \\
V_{E} & =-\frac{3 e^{2}}{R_{\mathrm{Fe}-\mathrm{As}}^{4}} \frac{8}{\sqrt{3}} x y \cdot \tilde{u}_{E}, \\
V_{66} & =\frac{3 e^{2}}{R_{\mathrm{Fe}-\mathrm{As}}^{4}} \sqrt{6}\left(x^{2}-y^{2}\right) \cdot \tilde{u}_{66},
\end{aligned}
$$

for both Fe-A and Fe-B sites, where $(x, y, z)$ is the coordinates of $d$-electron. $\tilde{\boldsymbol{u}}_{\phi} \equiv \boldsymbol{u}_{\phi}-\boldsymbol{u}_{\mathrm{Fe}}(\phi=44,66, E)$ is the relative displacements of the nearest As ions from the center Fe ion; $u_{\phi}\left(u_{\mathrm{Fe}}\right)$ is the displacement vector of the As- (Fe-) ion we are considering from the original position. Note that the shear strain tensors are given as $\epsilon_{44(E)}=\tilde{u}_{44(E)} /\left(a_{\mathrm{Fe}-\mathrm{Fe}} / 2\right)=2 \tilde{u}_{44(E)}$ and $\epsilon_{66}=\tilde{u}_{66} /\left(a_{\mathrm{Fe}-\mathrm{Fe}} / \sqrt{2}\right)=\sqrt{2} \tilde{u}_{66}$.

The corresponding operators in the ten-orbital model are respectively given as

$$
\begin{aligned}
& \hat{V}_{44}=-\frac{3 e^{2} a_{d}^{2}}{R_{\mathrm{Fe}-\mathrm{As}}^{4}} \frac{8}{7 \sqrt{3}} \hat{O}_{x z} \cdot \tilde{u}_{44}, \\
& \hat{V}_{E}=-\frac{3 e^{2} a_{d}^{2}}{R_{\mathrm{Fe}-\mathrm{As}}^{4}} \frac{8}{7 \sqrt{3}} \hat{O}_{x y} \cdot \tilde{u}_{E}, \\
& \hat{V}_{66}=\frac{3 e^{2} a_{d}^{2}}{R_{\mathrm{Fe}-\mathrm{As}}^{4}} \frac{2 \sqrt{6}}{7} \hat{O}_{x^{2}-y^{2}} \cdot \tilde{u}_{66} .
\end{aligned}
$$

Therefore, the acoustic modes in Fig. 7 (a)-(c) couple with the quadrupole susceptibilities at $\boldsymbol{q} \approx 0 ; \chi_{x z}^{Q}(0)$, $\chi_{x y}^{Q}(0)$, and $\chi_{x^{2}-y^{2}}^{Q}(0)$ for $\phi=44 E$, and 66 in the tenorbital model, respectively.

To study the softening in the five-orbital models, we have to perform the "unfold-gauge transformation" for Eqs. (23)-(25). Under the gauge transformation, Eqs. (24) and (25) are invariant, while Eq. (23) is changed to

$$
\hat{V}_{44}^{\prime}=\mp \frac{3 e^{2} a_{d}^{2}}{R_{\mathrm{Fe}-\mathrm{As}}^{4}} \frac{8}{7 \sqrt{3}} \hat{O}_{x z} \cdot \tilde{u}_{44},
$$

where the $-(+)$ sign corresponds to Fe-A $(\mathrm{Fe}-\mathrm{B})$ site. In the "five-orbital model", therefore, the softening of $C_{E}$ and $C_{66}$ are caused by $\chi_{x y}^{Q}(\mathbf{0}, 0)$ and $\chi_{x^{2}-y^{2}}^{Q}(\mathbf{0}, 0)$, respectively, while the softening of $C_{44}$ is caused by $\chi_{x z}^{Q}((\pi, \pi), 0)$. Therefore, the softening in shear modulus $\left(C_{66}\right.$ and $\left.C_{44}\right)$ does not occur within the $\mathrm{RPA}^{48}$.

Next, we derive the effective el-el interaction due to $\boldsymbol{k} \rightarrow 0$ transverse acoustic modes. In the case of $\boldsymbol{k}=$ 


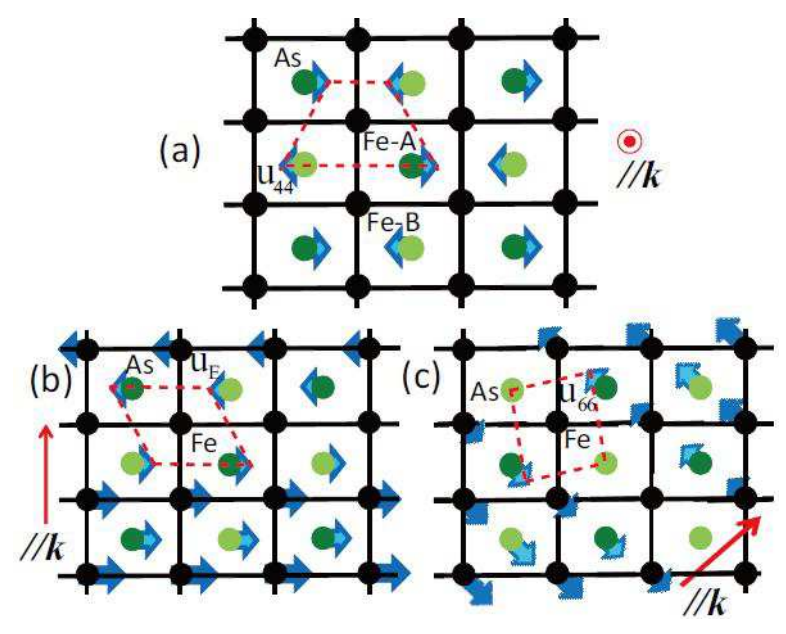

FIG. 7: (Color online) Displacement vectors $\boldsymbol{u}_{\mathrm{As}}$ and $\boldsymbol{u}_{\mathrm{Fe}}$ in the transverse acoustic modes that couple with (a) $C_{44}$, (b) $C_{E}$, and (c) $C_{66} . C_{66}$ mode corresponds to the orthorhombic structure transition.

$k \cdot(1,1) / \sqrt{2}$ and $k \ll 1$ shown in Fig. 7 (c), the displacement operator for the As site at $\boldsymbol{R}_{s}$ is

$$
u_{s}=\sum_{\boldsymbol{k}} \sqrt{\frac{1}{2 N M \omega_{\boldsymbol{k}}}}\left[a_{\boldsymbol{k}} e^{i \boldsymbol{k} \boldsymbol{R}_{s}}+a_{\boldsymbol{k}}^{\dagger} e^{-i \boldsymbol{k} \boldsymbol{R}_{s}}\right],
$$

where $a_{\boldsymbol{k}}$ and $a_{\boldsymbol{k}}^{\dagger}$ satisfy the commutation relation $\left[a_{\boldsymbol{k}}, a_{\boldsymbol{k}^{\prime}}^{\dagger}\right]=\delta_{\boldsymbol{k}, \boldsymbol{k}^{\prime}}, M$ is the mass of As-ion, and $\omega_{\boldsymbol{k}}=v_{\boldsymbol{k}}|\boldsymbol{k}|$; $v_{\boldsymbol{k}}$ is the bare acoustic phonon velocity. The Fourier transformation of $u_{s}$ is given as $u_{\boldsymbol{k}}=\sqrt{\frac{1}{2 M \omega_{k}}}\left(a_{\boldsymbol{k}}+a_{-\boldsymbol{k}}^{\dagger}\right)$. Then, the Fourier transformation of the acoustic-phonon Green function $D_{\boldsymbol{k}}(\tau) \equiv\left\langle T_{\tau} u_{\boldsymbol{k}}^{i}(\tau) u_{\boldsymbol{k}}^{i}(0)\right\rangle$ is

$$
D_{\boldsymbol{k}}\left(\omega_{n}\right)=\frac{2 \omega_{\boldsymbol{k}}}{\omega_{n}^{2}+\omega_{\boldsymbol{k}}^{2}}\left\langle u_{\boldsymbol{k}}^{2}\right\rangle_{0}
$$

where $\left\langle u_{\boldsymbol{k}}^{2}\right\rangle_{0}=\left(1 / 2 M \omega_{\boldsymbol{k}}\right)$.

As understood in Fig. 7 (c), the relative displacement with the origin at the Fe-ion, $\tilde{u}_{s}$, is given as $\frac{1}{2}\left(u_{s^{\prime}}-u_{s}\right)$, where $\boldsymbol{R}_{s^{\prime}}=(0.5,0.5)$ and $\boldsymbol{R}_{s}=(-0.5,-0.5)$ with the origin at the Fe-ion. Considering that $\left(\boldsymbol{R}_{s^{\prime}}-\boldsymbol{R}_{s}\right) \cdot \boldsymbol{k}=$ $\sqrt{2} k$, its Fourier transformation is given as

$$
\tilde{u}_{\boldsymbol{k}} \equiv \sum_{s} \tilde{u}_{s} e^{-i \boldsymbol{k} \boldsymbol{R}_{s}} \sim \frac{i k}{\sqrt{2}} u_{\boldsymbol{k}}
$$

To calculate the shear modulus, we need the quadrupole susceptibility in the " $k$-limit", in which we put $\omega=0$ first, and take the limit $k \rightarrow 0$ later $^{49}$. For this purpose, we derive the effective el-el interaction due to the transverse acoustic phonon in the $k$-limit. The el-el interaction due to the phonon in Fig. 7 (c) is given by the second-order term of Eq. (25). Using the relation
$D_{\boldsymbol{k}}(0) \boldsymbol{k}^{2}=2\left(1 / 2 M \omega_{\boldsymbol{k}}\right) \boldsymbol{k}^{2} / \omega_{\boldsymbol{k}}=1 / M v_{\boldsymbol{k}}^{2}$, it is given as

$$
\begin{aligned}
H_{66}= & -g_{66} \sum_{\boldsymbol{k \boldsymbol { k } ^ { \prime } , l l ^ { \prime } m m ^ { \prime } , \sigma \sigma ^ { \prime }}} o_{x^{2}-y^{2}}^{l l^{\prime}} o_{x^{2}-y^{2}}^{m m^{\prime}} \\
& \times c_{l \boldsymbol{k} \sigma}^{\dagger} c_{l^{\prime} \boldsymbol{k} \sigma} c_{m \boldsymbol{k}^{\prime} \sigma^{\prime}}^{\dagger} c_{m^{\prime} \boldsymbol{k}^{\prime} \sigma^{\prime}} \\
g_{66}= & \frac{B^{2}}{R_{F e-A s}^{2}} \frac{1}{M v_{\boldsymbol{k}}^{2}},
\end{aligned}
$$

where $B \equiv \frac{3 e^{2}}{R_{\mathrm{Fe}-\mathrm{As}}}\left(\frac{a_{d}}{R_{\mathrm{Fe}-\mathrm{As}}}\right)^{2} \frac{2 \sqrt{3}}{7} ; B=0.95 \mathrm{eV}$ for $a_{d}=0.77 \AA$ (=Shannon crystal radius of $\mathrm{Fe}^{2+}$ ) and $R_{\mathrm{Fe}-\mathrm{As}}=2.4 \AA$. Therefore,

$$
g_{66}=\eta_{66}^{2} C_{66,0}^{-1},
$$

where $C_{66,0} \equiv M v_{\boldsymbol{k}}^{2}$ is the bare shear modulus, and $\eta_{66}=$ $B R_{\mathrm{Fe}-\mathrm{As}}^{-1}$ is the quadrupole-strain coupling constant. If we put $v_{\boldsymbol{k}} \sim 0.024 \mathrm{eV} \AA\left(v_{\boldsymbol{k}} \sim 0.018 \mathrm{eV} \AA\right)$ according to the first principle study ${ }^{50}$, we obtain $g_{66}=0.12 \mathrm{eV}\left(g_{66}=\right.$ $0.21 \mathrm{eV}$ ). On the other hand, we obtain $g=0.34 \mathrm{eV}$ for the Fe-ion optical phonons with $\omega_{\mathrm{D}}=0.02 \mathrm{eV}^{10}$. Thus, $g_{66} / g=1 / 2 \sim 1 / 3$ in the present point charge model.

In the same way, we also derive the el-el interactions due to the acoustic phonon with $\boldsymbol{k}=k \cdot(0,1)$, shown in Fig. 7 (b). For this mode, the relative displacement $\tilde{u}_{\boldsymbol{k}}$ is given as $\tilde{u}_{s} \equiv \frac{1}{2}\left(u_{s^{\prime}}-u_{s}\right)$ with $\boldsymbol{R}_{s^{\prime}}=(0.5,0.5)$ and $\boldsymbol{R}_{s}=(0.5,-0.5)$. Since $\left(\boldsymbol{R}_{s^{\prime}}-\boldsymbol{R}_{s}\right) \cdot \boldsymbol{k}=k$, its Fourier transformation is given by

$$
\bar{u}_{\boldsymbol{k}} \equiv \sum_{s} \tilde{u}_{s} e^{-i \boldsymbol{k} \boldsymbol{R}_{s}} \sim \frac{i k}{2} u_{\boldsymbol{k}} .
$$

Then, the phonon-mediated el-el interactions are given by the second-order terms of Eq. (24). As a result, the el-el interactions due to phonons in Fig. 7 (b) is given as

$$
\begin{aligned}
H_{E}= & -g_{E} \sum_{\boldsymbol{k \boldsymbol { k } ^ { \prime } , l l ^ { \prime } m m ^ { \prime } , \sigma \sigma ^ { \prime }}} o_{x y}^{l l^{\prime}} o_{x y}^{m m^{\prime}} \\
& \times c_{l \boldsymbol{k} \sigma}^{\dagger} c_{l^{\prime} \boldsymbol{k} \sigma} c_{m \boldsymbol{k}^{\prime} \sigma^{\prime}}^{\dagger} c_{m^{\prime} \boldsymbol{k}^{\prime} \sigma^{\prime}},
\end{aligned}
$$

where $g_{E}=\frac{B^{\prime 2}}{R_{F e-A s}^{2}} \frac{1}{M v_{\boldsymbol{k}}^{2}}$, and $B^{\prime} \equiv$ $\frac{3 e^{2}}{R_{\mathrm{Fe}-\mathrm{As}}}\left(\frac{a_{d}}{R_{\mathrm{Fe}-\mathrm{As}}}\right)^{2} \frac{4}{7 \sqrt{3}}$. In the same way, we obtain $g_{44}=g_{E}$. Therefore, $g_{\phi}=\eta_{\phi}^{2} C_{\phi, 0}^{-1}$ and $\eta_{\phi}^{2}=0.44 \eta_{66}^{2}$ for $\phi=44, E$. In conclusion, $g_{E}=g_{44}=0.44 g_{66}$ if $v_{\boldsymbol{k}}$ is equivalent for all modes.

\section{SOFTENING OF SHEAR MODULI}

\section{A. Softening due to one-orbiton process; the RPA}

Here, we calculate the shear modulus given by the oneorbiton process using the RPA. For this purpose, we introduce the following shear modulus susceptibilities in 
the five-orbital model, in the absence of $e$-ph interaction due to $\boldsymbol{q} \approx 0$ acoustic phonon:

$$
\begin{aligned}
\chi_{E} & =2 \chi_{x y}^{Q}(\mathbf{0}, 0), \\
\chi_{44} & =2 \chi_{x z}^{Q}((\pi, \pi), 0), \\
\chi_{66} & =2 \chi_{x^{2}-y^{2}}^{Q}(\mathbf{0}, 0),
\end{aligned}
$$

where the factor 2 comes from the spin degeneracy. They are schematically depicted in Fig. 8 (a). Note that $\chi_{44}=$ $2 \chi_{x z}^{Q}(\mathbf{0}, 0)$ in the ten-orbital model. According to Sec. 2 in Ref. 51, the shear modulus is given by the second derivative of the Free energy with respect to the shear strain tensor: The expression for the shear modulus $C_{\phi}$ $(\phi=E, 44,66)$ is ${ }^{51,52}$

$$
C_{\phi}=C_{\phi, 0}-\eta_{\phi}^{2} \chi_{\phi},
$$

where $C_{\phi, 0}=v_{\phi}^{2} \rho$ is the bare shear modulus, where $v_{\phi}$ is the bare acoustic phonon velocity and $\rho$ is the mass density. $\eta_{\phi}$ is the quadrupole-strain coupling constant due to the "acoustic phonon" given in Sec. IV. In Eq. (38), the condition for the structure transition, $C_{\phi}=0$, is satisfied when $\chi_{\phi}=g_{\phi}^{-1}(\gg 1)$. That is, the structure transition occurs prior to the divergence of $\chi_{\phi}$.

We can rewrite the expression for $C_{\phi}$ given in Eq. (38) as follows:

$$
\begin{aligned}
C_{\phi}^{-1} & =C_{\phi, 0}^{-1}\left[1+g_{\phi} \tilde{\chi}_{\phi}\right], \\
\tilde{\chi}_{\phi} & =\chi_{\phi} /\left(1-g_{\phi} \chi_{\phi}\right),
\end{aligned}
$$

where $g_{\phi} \equiv \eta_{\phi}^{2} C_{\phi, 0}^{-1}$ is the effective el-el interaction due to acoustic phonon given in the previous section. In Eq. (39), the condition $C_{\phi}=0$ corresponds to the divergence of $\tilde{\chi}_{\phi}$, since $\tilde{\chi}_{\phi}$ is the total susceptibility including the $e$-ph interactions due to acoustic phonons.

If we put $U=0$ for simplicity, Eq. (40) is expressed as

$$
\begin{aligned}
\tilde{\chi}_{44} & =\chi_{44}^{0} /\left(1-\left(g+g_{44}\right) \chi_{44}^{0}\right), \\
\tilde{\chi}_{E} & =\chi_{E}^{0} /\left(1-\left(g+g_{E}\right) \chi_{E}^{0}\right), \\
\tilde{\chi}_{66} & =\chi_{66}^{0} /\left(1-g_{66} \chi_{66}^{0}\right),
\end{aligned}
$$

where the suffix 0 represents the bare susceptibility. According to $\tilde{\chi}_{44}$ in Eq. (41), $g$ in Eq. (19) for $\chi_{x z}^{Q}$ is replaced with $g+g_{44}$ when both optical and acoustic phonons are taken into account. Therefore, we have to reduce $g$ to $g-g_{44}$ to keep the charge Stoner factor $\alpha_{c}$ and $\chi_{x z}^{Q}(\boldsymbol{Q})$ unchanged. Considering the relation $g_{44} \sim g_{E}$ that we derived in the previous section, we obtain $\tilde{\chi}_{E} \sim \chi_{E}$. Then, we conclude that (i) $C_{44} \sim C_{44,0}$ since $\chi_{44}$ is seldom enhanced in the RPA. Also, (ii) $C_{E}$ softens to some extent since $\chi_{x y}^{Q}(\mathbf{0})$ is weakly enhanced as shown in Fig. 5, although the relation $C_{E}=0$ will not be satisfied because of the relation $\tilde{\chi}_{E} \sim \chi_{E}$.

As for $C_{66}$ given in Eq.(43), $\chi_{66}^{0}$ in the present model is $\sim 2 \mathrm{eV}^{-1}$, while we estimate $g_{66}=0.1 \sim 0.2 \mathrm{eV}$. Therefore, we expect (iii) $C_{66} \sim C_{66,0}$ in the RPA, which is inconsistent with experimentally observed large softening in $C_{66}{ }^{31}$. In the RPA, the softening in shear modulus
$\left(C_{66}, C_{44}\right.$ and $\left.C_{E}\right)$ is small according to Eqs. (35)-(37) and Figs. 4 (a)-(c). In the next subsection, we analyze $\chi_{66}$ by taking account of the two-orbiton process that is not included in the RPA.

(a)
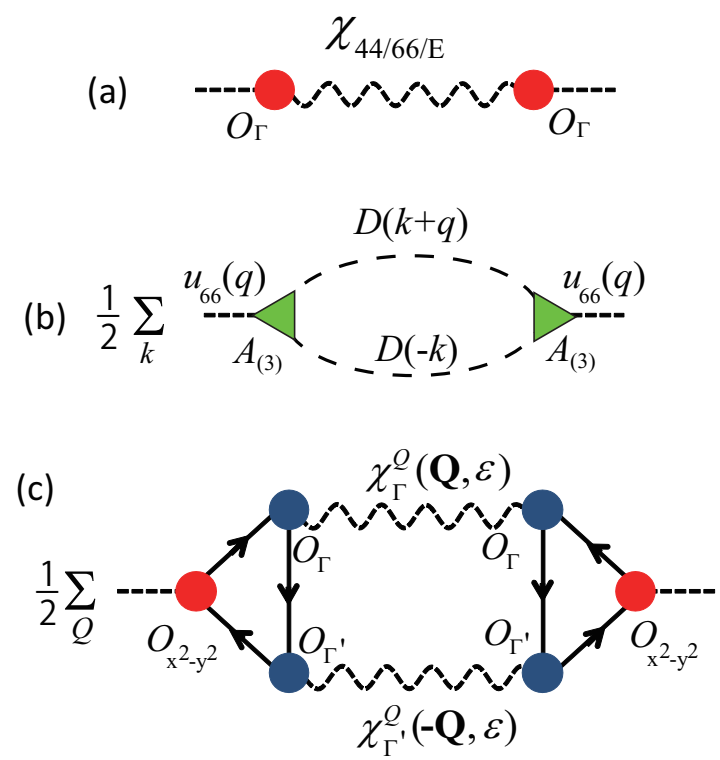

(d)
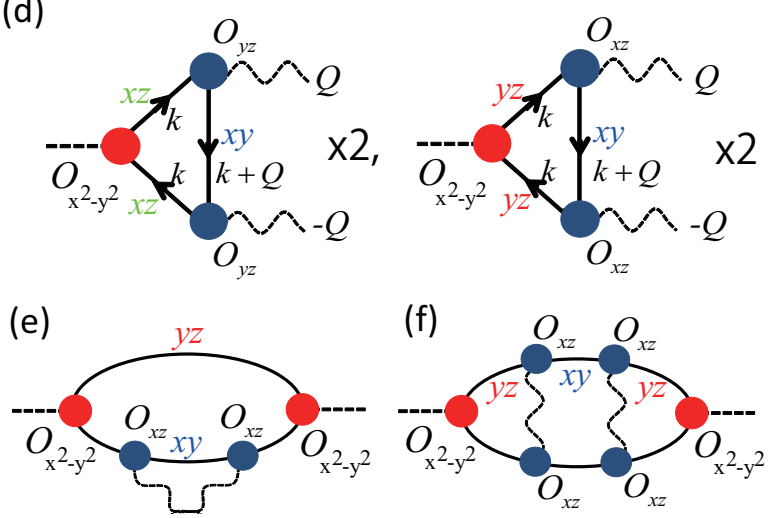

FIG. 8: (Color online) (a) Diagrammatic expression for the shear modulus susceptibilities $\chi_{\phi}(\phi=44,66, E)$ in the RPA. (b) The second-order term with respect to the third-order anharmonic phonon-phonon interaction $A_{(3)} u_{x}^{2} u_{66}$. This diagram represents the virtual process in which an acoustic phonon with $\boldsymbol{q}=0$ breaks into two optical phonons conserving the total momentum. (c) Two-orbiton term for the shear modulus susceptibilities $\chi_{66}^{T O}$, which is the irreducible susceptibility of $\chi_{x^{2}-y^{2}}(\mathbf{0}, 0)$. This term gives the softening of $C_{66}$. (d) Dominant contribution for the three-point vertex $A_{x^{2}-y^{2}}(\Gamma, \Gamma ; \boldsymbol{q})$ for $\Gamma=x z$ and $y z$. (e) Self-energy correction due to $\chi_{x z}^{Q}(q)$. (f) Second-order Maki-Thompson-type vertex correction with respect to $\chi_{x z}^{Q}(q)$.

\section{B. Softening due to two-orbiton process; the Aslamazov-Larkin-type diagram}

In the previous subsection, we studied the softening of shear moduli within the RPA. However, the obtained 
softening is very small since only the AFQ fluctuations develop in the present model. In this subsection, we analyze $\chi_{66}$ by taking account of the "two-orbiton process" that is not included in the RPA. In usual cases, this higher order process is negligible. However, it gives divergent increase in $\chi_{66}$, and creates the FQ-QCP near the AFQ-QCP. For this reason, the orthorhombic structure transition $\left(C_{66}=0\right)$ is induced by the two-orbiton process.

Before calculating the two-orbiton process, let us consider the third order anharmonic phonon-phonon coupling $\sim A_{(3)} u_{x}^{2} u_{66}$, where $u_{x}$ is the displacement of Feion optical mode in Eq. (2), and $u_{66}$ is the displacement of As-ion acoustic node in Eq. (22) or (25). Figure 8 (b) shows the second-order-term with respect to $A_{(3)}$, where $D(q)$ is the optical phonon Green function in Eq. (9), and the factor $1 / 2$ is introduced to cancel the overcounting with respect to the upside-down diagrams. This term gives a self-energy correction for the acoustic phonon Green function. This two phonon process would be measurable in Raman spectroscopy. By considering the $e$-ph interaction, each $D(q)$ in (b) is replaced with $D(q)+(\eta D(q))^{2} \cdot \chi_{y z}^{Q}(q)$.

Here, we study the ferro-quadrupole susceptibility due to the "two-orbiton term" given by Fig. 8 (c), and analyze for the softening of $C_{66}$. Since the coefficient of the anharmonic phonon-phonon coupling $A_{(3)}$ would be small in iron pnictides, we instead consider the threepoint vertex given by electron Green functions in Fig. 8 (d). The two-orbiton term in Fig. 8 (c) is similar to the Aslamazov-Larkin (AL) term for the excess conductivities due to superconducting fluctuations; such as longitudinal ${ }^{53}$ and Hall ${ }^{54}$ conductivities, and the Nernst coefficient ${ }^{55}$. Figure 8 (c) is a virtual process in which one $O_{x^{2}-y^{2}}$-type orbiton with $\boldsymbol{q}=0$ breaks into two $O_{x z}$-type orbitons with zero total momentum $( \pm \boldsymbol{Q})$. After taking the momentum summation, the two-orbiton term (c) in $2 \mathrm{D}$ systems would be strongly enhanced for $\boldsymbol{q}=0$ since $\chi_{y z}^{Q}(\boldsymbol{k})$ has a large peak at finite momentum $\boldsymbol{k}$. The mathematical expression for the two-orbiton process in Fig. 8 (c) for $\chi_{66}$, which we call $\chi_{66}^{\mathrm{TO}}$, is given as

$$
\begin{aligned}
\chi_{66}^{\mathrm{TO}}= & \frac{1}{2}(2 g)^{2} T \sum_{\boldsymbol{q}, l, \Gamma, \Gamma^{\prime}} A_{x^{2}-y^{2}}\left(\Gamma, \Gamma^{\prime} ; \boldsymbol{q}, \omega_{l}\right) A_{x^{2}-y^{2}}\left(\Gamma, \Gamma^{\prime} ; \boldsymbol{q},\right. \\
& \times\left(1+2 g \chi_{\Gamma}^{Q}\left(\boldsymbol{q}, \omega_{l}\right)\right)\left(1+2 g \chi_{\Gamma^{\prime}}^{Q}\left(\boldsymbol{q}, \omega_{l}\right)\right) \\
\approx & \frac{1}{2}(2 g)^{4} T \sum_{\boldsymbol{q}, l, \Gamma, \Gamma^{\prime}} A_{x^{2}-y^{2}}\left(\Gamma, \Gamma^{\prime} ; \boldsymbol{q}, \omega_{l}\right) A_{x^{2}-y^{2}}\left(\Gamma, \Gamma^{\prime} ; \boldsymbol{q},\right. \\
& \times \chi_{\Gamma}^{Q}\left(\boldsymbol{q}, \omega_{l}\right) \chi_{\Gamma^{\prime}}^{Q}\left(\boldsymbol{q}, \omega_{l}\right),
\end{aligned}
$$

where $A_{x^{2}-y^{2}}\left(\Gamma, \Gamma^{\prime} ; \boldsymbol{q}, \omega_{l}\right)$ is the three point vertex with respect to $\hat{V}_{66}$ in Eq. (25) and quadrupole operators $\hat{O}_{\Gamma}$ and $\hat{O}_{\Gamma^{\prime}}$, shown in Fig. $8(\mathrm{~d})$. We used the relation $\chi_{\Gamma}^{Q}(q)=\chi_{\Gamma}^{Q}(-q)$. When $U=0, A_{x^{2}-y^{2}}$ for $\omega_{l}=0$ is given as

$$
\begin{aligned}
A_{x^{2}-y^{2}}\left(\Gamma, \Gamma^{\prime} ; \boldsymbol{q}\right)= & -2 T \sum_{n, \boldsymbol{k}} \operatorname{Tr}\left\{\hat{G}_{\boldsymbol{k}}\left(\epsilon_{n}\right) \hat{o}_{x^{2}-y^{2}} \hat{G}_{\boldsymbol{k}}\left(\epsilon_{n}\right)\right. \\
& \left.\times \hat{o}_{\Gamma} \hat{G}_{\boldsymbol{k}+\boldsymbol{q}}\left(\epsilon_{n}\right) \hat{o}_{\Gamma^{\prime}}\right\}
\end{aligned}
$$

where the factor 2 in front of Eq. (45) accounts for the diagrams with reversing three Green functions $\hat{G}_{\boldsymbol{k}}\left(\epsilon_{n}\right) \rightarrow$ $\hat{G}_{-\boldsymbol{k}}\left(-\epsilon_{n}\right)$. Near the QCP $g \lesssim g_{c}$, the most divergent quadrupole susceptibility is $\chi_{x z(y z)}^{Q}$. Therefore, the dominant contribution for $\chi_{66}^{\mathrm{TO}}$ in Eq. (44) will be given by the term with $\Gamma=\Gamma^{\prime}=x z$ or $y z$. After the analytic continuation, the functional form of $\chi_{x z / y z}^{Q}(\boldsymbol{q}, \omega)$ for $\boldsymbol{q} \approx \boldsymbol{Q}_{x z}=(\pi, 0)$ or $\boldsymbol{q} \approx \boldsymbol{Q}_{y z}=(0, \pi)$ would be approximately given as

$$
\chi_{\Gamma}^{Q}(\boldsymbol{q}, \omega+i \delta)=\frac{c \xi^{2}}{1+\xi^{2}\left(\boldsymbol{q}-\boldsymbol{Q}_{\Gamma}\right)^{2}-i \omega / \omega_{0}},
$$

for $\Gamma=x z$ or $y z$, where $\xi$ is the correlation length and $\omega_{0}$ is the characteristic energy of the fluctuation. The relation $\xi^{2} \propto \omega_{0}^{-1}$ holds in the RPA.

Next, we consider the temperature dependence of $\xi$. In the FLEX approximation ${ }^{11}$ or SCR theory ${ }^{56}$, the bare susceptibility $\chi_{\phi}^{0}$ is approximately suppressed as $\chi_{\phi}^{0}-\alpha T$ $(\alpha>0)$ due to the thermal fluctuations, which are described as the self-energy and Maki-Thompson vertex corrections. In this case, we obtain $\chi_{x z}^{Q}(\boldsymbol{Q}, 0) \propto$ $\left(1-g \chi_{x z}^{0}(\boldsymbol{Q}, 0)+g \alpha T\right)^{-1} \propto\left(T-T_{\mathrm{AFQ}}\right)^{-1}$ based on the RPA, where $T_{\mathrm{AFQ}}=-\left(1-g \chi_{x z}^{0}(\boldsymbol{Q}, 0)\right) / g \alpha$ is the transition temperature to the AFQ ordered state. Since $\chi_{x z}^{Q}(\boldsymbol{Q}, 0) \propto \xi^{2}$, we assume the following relations

$$
\begin{aligned}
\xi^{2} & =l\left(T-T_{\mathrm{AFQ}}\right)^{-1}, \\
\omega_{0} & =l^{\prime}\left(T-T_{\mathrm{AFQ}}\right),
\end{aligned}
$$

where $l, l^{\prime}$ are constants. Note that $\omega_{0} \xi^{2}$ is temperature independent ${ }^{56}$. By carrier doping, $T_{\mathrm{AFQ}}$ changes from positive to negative, while other model parameters $(c, l$, and $l^{\prime}$ ) would be insensitive to doping. As shown in Fig. $5, \chi_{x z}^{Q}(\boldsymbol{Q}, 0) \sim 2.4 \times\left(1-\alpha_{c}\right)^{-1} \sim 12 \xi^{2}$. In the case (i) $T_{\mathrm{AFQ}}>0$, the relation $\omega_{0}<T$ is satisfied near $T_{\mathrm{AFQ}}$. In the opposite case (ii) $T_{\mathrm{AFQ}}<0$, the relation $\omega_{0}>$ wit) will hold for wide range of temperatures. Note that the present phenomenological model in Eqs. (46)-(48) is reproduced by the microscopic calculation by FLEX approximation $^{11}$. As for the spin propagator in cuprate $\omega$ s $)$ uperconductors, the relation $\omega_{0}>T\left(\omega_{0}<T\right)$ holds in over-doped (under-doped) systems.

Here, we comment on the self-energy correction and (44) Maki-Thompson-type vertex correction for $\chi_{66}^{\mathrm{TO}}$, shown in Figs. 8 (e) and (f) respectively. The former term is included in the FLEX approximation, and it gives the Curie-Weiss behavior of $\chi_{x z}^{Q}(\boldsymbol{Q}, 0)$ given by Eqs. (46)(48), as reported in Ref. 11 or Ref. 56. The latter term would be negligible since its temperature dependence is smaller than that of the former term. For this reason, 
we concentrate on the two-orbiton term in Fig. 8 (c) hereafter.

From now on, we perform the numerical calculation of the two-orbiton process in the case (i), in which the relations $\xi \gg 1$ and $\omega_{0} \ll T$ are realized near the orbitalordered state. In this case, the dominant contribution in Eq. (44) comes form the terms with $\Gamma=\Gamma^{\prime}=x z$ and $y z$. Also, we can safely apply the classical approximation, in which the terms with $\omega_{l} \neq 0$ are dropped in Eq. (44). Under these approximations, Eq. (44) is simplified as

$$
\chi_{66}^{\mathrm{TO}}=(2 g)^{4} T \sum_{\boldsymbol{q}}\left\{A_{x^{2}-y^{2}}(x z, x z ; \boldsymbol{q}) \chi_{x z}^{Q}(\boldsymbol{q}, 0)\right\}^{2},(49)
$$

To calculate $A_{x^{2}-y^{2}}\left(\Gamma, \Gamma^{\prime} ; \boldsymbol{q}\right)$, we introduce a uniform FQ potential term $H^{\prime}=h \sum_{i} \hat{O}_{x^{2}-y^{2}}^{i}$, where $h$ is an infinitesimal small constant. Then, the three point vertex is given as the following Ward identity ${ }^{57}$ :

$$
A_{x^{2}-y^{2}}\left(\Gamma, \Gamma^{\prime} ; \boldsymbol{q}\right)=\frac{1}{h}\left[\bar{\chi}_{\Gamma, \Gamma^{\prime}}^{Q}(\boldsymbol{q}, 0 ; h)-\bar{\chi}_{\Gamma, \Gamma^{\prime}}^{Q}(\boldsymbol{q}, 0 ; 0)\right],(50)
$$

where $\bar{\chi}_{\Gamma, \Gamma^{\prime}}^{Q}\left(\boldsymbol{q}, \omega_{l} ; h\right)$ is the "irreducible" quadrupole susceptibility with respect to $g$. In the numerical calculation, we have to fix $\mu$ against the change in $h$. Equation (50) gives the correct three-point vertex even for $U \neq 0$. In the case of $U=0, \bar{\chi}_{\Gamma, \Gamma^{\prime}}^{Q}$ is simply given as $\bar{\chi}_{\Gamma, \Gamma^{\prime}}^{Q}\left(\boldsymbol{q}, \omega_{l} ; h\right)=-T \sum_{\boldsymbol{k}, n} \operatorname{Tr}\left\{\hat{o}_{\Gamma} \hat{G}\left(\boldsymbol{k}+\boldsymbol{q}, \epsilon_{n}+\right.\right.$ $\left.\left.\omega_{l} ; h\right) \hat{o}_{\Gamma^{\prime}} \hat{G}\left(\boldsymbol{k}, \epsilon_{n} ; h\right)\right\}$. The obtained $A_{x^{2}-y^{2}}(x z, x z ; \boldsymbol{q})$ for $U=0$ is presented in Fig. 9 (a). Similar result is obtained for $U=0.8 \mathrm{eV}$.

According to the functional form of $\chi_{x z}^{Q}(\boldsymbol{q}, 0)$ in Eq. (46), $\chi_{66}^{\mathrm{TO}} / T \propto \sum_{\boldsymbol{q}}\left\{\chi_{x z}^{Q}(\boldsymbol{q}, 0)\right\}^{2} \propto \xi^{2} \propto\left(T-T_{\mathrm{AFQ}}\right)^{-1}$ in two-dimensional systems. The numerical result for $\chi_{66}^{\mathrm{TO}} / T$ given in Eq. (49) is shown in Fig. 9 (b). The obtained result follows the relation $\chi_{66}^{\mathrm{TO}} / T \sim 0.1(1-$ $\left.\alpha_{c}\right)^{-1}$. Since $6 \xi^{2} \sim\left(1-\alpha_{c}\right)^{-1}$, the relation $\chi_{66}^{\mathrm{TO}} / T \sim$ $0.6 \xi^{2}$ is verified numerically.

In the same way, two-orbiton processes for other two shear modulus susceptibilities, $\chi_{44}^{\mathrm{TO}}$ and $\chi_{E}^{\mathrm{TO}}$, are proportional to the square of $A_{\Gamma}(x z, x z ; \boldsymbol{q})$ for $\Gamma=x z$ and $x y$, respectively. However, they are four orders of magnitude smaller than $\chi_{66}^{\mathrm{TO}}$, as recognized in TABLE I: This selection rule for $A_{\Gamma}$ is understood as follows: According to the relation $\operatorname{Tr}\left\{\hat{O}_{\Gamma} \hat{O}_{x z} \hat{O}_{x z}\right\}=0$ for $\Gamma=x z, x y$, we recognize that $A_{x z / x y}$ originates from the off-diagonal terms of the Green function $G_{l, m}(l \neq m)$ that is much smaller than the diagonal terms. For this reason, the two-orbiton process is negligible except for $\chi_{66}^{\mathrm{TO}}$.

Here, we discuss the softening of $C_{66}$ by taking the twoorbiton process into account: According to Eqs. (39) and (40), we obtain

$$
\begin{aligned}
C_{66}^{-1} & =C_{66,0}^{-1}\left[1+g_{66} \tilde{\chi}_{66}\right], \\
\tilde{\chi}_{66} & =\frac{a_{66}+\chi_{66}^{\mathrm{TO}}}{1-g_{66}\left(a_{66}+\chi_{66}^{\mathrm{TO}}\right)},
\end{aligned}
$$

where $a_{66} \equiv 2 \chi_{66}^{0}(\mathbf{0}, 0)$. Now, we consider the case (i) $T_{\mathrm{AFQ}}>0$ and $\omega_{0} \ll T$. As we have obtained the relation

\begin{tabular}{c||c|c|c|c}
\hline$\Gamma$ & $x^{2}-y^{2}$ & $x z$ & $y z$ & $x y$ \\
\hline$A_{\Gamma}(x z, x z, \boldsymbol{Q})$ & -0.60 & $1.9 \times 10^{-3}$ & $-1.0 \times 10^{-3}$ & $3.2 \times 10^{-4}$ \\
\hline
\end{tabular}

TABLE I: Three point vertex $A_{\Gamma}(x z, x z, \boldsymbol{Q})$ for $\Gamma=x^{2}-y^{2}$, $x z, y z$, and $x y . \quad \boldsymbol{Q}=(\pi, 0)$ corresponds to the peak position of $\chi_{x z}^{Q}(\boldsymbol{q}, 0)$ in the five-orbital model. We recognize that $A_{\Gamma}(x z, x z, \boldsymbol{Q}) \sim O(1)$ only for $\Gamma=x^{2}-y^{2}$; This selection rule means that $\chi_{44, E}^{\mathrm{TO}} \ll 1$.

$\chi_{66}^{\mathrm{TO}} \propto T \xi^{2}$, we put $\chi_{66}^{\mathrm{TO}}=b_{66} T /\left(T-T_{\mathrm{AFQ}}\right)$. Since the temperature dependence of $a_{66}$ is small, we obtain

$$
\begin{aligned}
\tilde{\chi}_{66} & =\frac{a_{66}+b_{66}}{1-g_{66}\left(a_{66}+b_{66}\right)} \frac{T-\left(a_{66} /\left(a_{66}+b_{66}\right)\right) T_{\mathrm{AFQ}}}{T-T_{S}}, \\
T_{S} & =T_{\mathrm{AFQ}} \frac{1-g_{66} a_{66}}{1-g_{66}\left(a_{66}+b_{66}\right)}\left(>T_{\mathrm{AFQ}}\right) .
\end{aligned}
$$

Then, the difference between $T_{S}$ and $T_{\mathrm{AFQ}}$, which is conventionally denoted as $E_{\mathrm{JT}}{ }^{51,52}$, is given by $E_{\mathrm{JT}}=$ $T_{S}\left(g_{66} b_{66}\right) /\left(1-g_{66} a_{66}\right)>0$. According to Eq. (53), Eq. (51) is rewritten as

$$
C_{66}=C_{66,0}\left(1-g_{66}\left(a_{66}+b_{66}\right)\right) \frac{T-T_{S}}{T-T_{\mathrm{AFQ}}},
$$

Here, $g_{66}=0.1 \sim 0.2 \mathrm{eV}$ and $a_{66} \sim 2 \mathrm{eV}^{-1}$. We stress that Eqs. (53)-(55) are valid only for $\omega_{0} \ll T$.

Finally, we calculate the two-orbiton process analytically for general value of $\omega_{0} / T$. Since we cannot apply the classical approximation that was used to derive Eq. (49), we have to perform the analytic continuation ${ }^{57}$ of Eq. (44). The obtained expression including the quantum fluctuation contribution is given as

$$
\begin{aligned}
\chi_{66}^{\mathrm{TO}}= & (2 g)^{4}\left\{A_{x^{2}-y^{2}}(x z, x z ; \boldsymbol{Q})\right\}^{2} \sum_{\boldsymbol{q}} \int_{-\infty}^{\infty} \frac{d x}{\pi} n(x) \\
& \times 2 \operatorname{Im} \chi_{x z}^{Q}(\boldsymbol{q}, x+i \delta) \operatorname{Re} \chi_{x z}^{Q}(\boldsymbol{q}, x+i \delta),
\end{aligned}
$$

where $n(x)=\left(e^{\beta x}-1\right)^{-1}$ is the Bose distribution function, and we put $A_{x^{2}-y^{2}}$ outside of the $\boldsymbol{q}$-summation since its momentum dependence is much smaller than that of $\chi_{x z}^{Q}$. First, we perform the $x$-integration using the following equations:

$$
\begin{aligned}
& \frac{1}{e^{x}-1}=\sum_{n=1}^{\infty} \frac{2 x}{(2 n \pi)^{2}+x^{2}}+\frac{1}{x} \\
& \int_{-\infty}^{\infty} \frac{x^{2}}{\left(a^{2}+x^{2}\right)\left(b^{2}+x^{2}\right)^{2}} d x=\frac{\pi}{2 b(a+b)^{2}},
\end{aligned}
$$

where $a, b>0$. Then, the expression after the $x$ integration in Eq. (56) is given as

$$
c^{2} \xi^{4}\left(\omega_{0}^{2} T \sum_{n=1}^{\infty}\left(B_{\boldsymbol{q}} \omega_{0}+2 n \pi T\right)^{-2}+\frac{T}{2 B_{\boldsymbol{q}}^{2}}\right),
$$




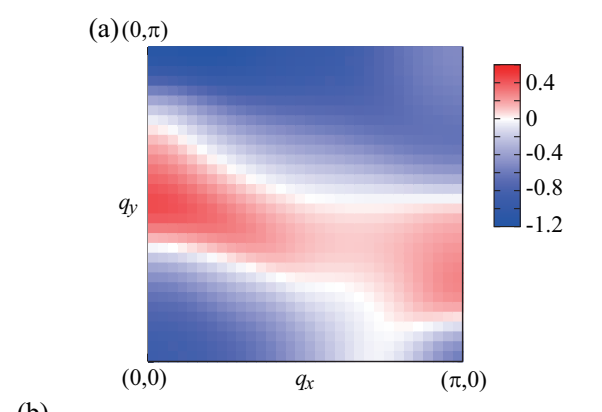

(b)

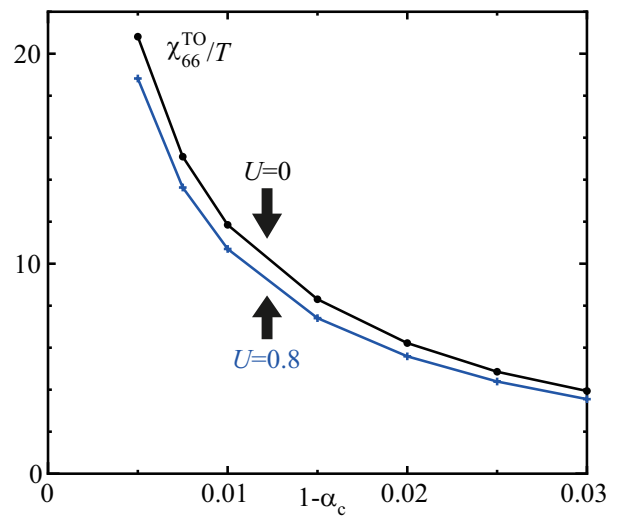

FIG. 9: (Color online) (a) Obtained $A_{x^{2}-y^{2}}(x z, x z ; \boldsymbol{q})$ for $U=$ 0 . We put $n=6.05$ and $T=0.05$. (b) $\chi_{66}^{\mathrm{TO}} / T$ given by Eq. (49) for $U=0$ and $U=0.8$ as function of $\alpha_{c}$. Using the relation $6 \xi^{2} \sim\left(1-\alpha_{c}\right)^{-1}$ given in the caption of Fig. 4 , we obtain $\chi_{66}^{\mathrm{TO}} / T \sim 0.1\left(1-\alpha_{c}\right)^{-1} \sim 0.6 \xi^{2}$. where $B_{\boldsymbol{q}}=1+\xi^{2}(\boldsymbol{q}-\boldsymbol{Q})^{2}$. Next, we take the $\boldsymbol{q}$ summation $\sum_{\boldsymbol{q}} \approx \frac{1}{2 \pi} \int_{0}^{\pi} q d q$ under the assumption $\xi^{2} \gg$ 1. Then, the $\boldsymbol{q}$-summation of the second term in Eq. (59) is easily obtained as $c^{2} \xi^{2} T / 8$. Also, the $\boldsymbol{q}$-summation of the first term is given as

$$
\begin{aligned}
& \frac{c^{2} \xi^{2} \omega_{0}}{8 \pi} \sum_{n=1}^{n_{\max }} \frac{1}{n+\frac{\omega_{0}}{2 \pi T}} \\
& =\frac{c^{2} \xi^{2} \omega_{0}}{8 \pi}\left[\psi\left(n_{\max }+\frac{\omega_{0}}{2 \pi T}+1\right)-\psi\left(\frac{\omega_{0}}{2 \pi T}+1\right)\right],
\end{aligned}
$$

where $\psi(x)$ is di-Gamma function, and the cutoff $n_{\max } \equiv$ $\left(1+\xi^{2} \pi^{2}\right) \omega_{0} / 2 \pi T$ originates from the fact that the $\boldsymbol{q}$ summation is limited to the region $|\boldsymbol{q}| \leq \pi$ in periodic systems. As a result, the final expression for the twoorbiton term is

$$
\chi_{66}^{\mathrm{TO}}=X \xi^{2}\left\{\frac{\omega_{0}}{\pi}\left[\psi\left(n_{\max }+\frac{\omega_{0}}{2 \pi T}+1\right)-\psi\left(\frac{\omega_{0}}{2 \pi T}+1\right)\right]+T\right\}
$$

where $X \equiv \frac{(2 g)^{4} c^{2}}{4 \pi}\left\{A_{x^{2}-y^{2}}(x z, x z ; \boldsymbol{Q})\right\}^{2}$. Here, we verify Eq. (61) in the opposite two limits: In the case (i) $\omega_{0} \ll T$, the di-Gamma functions in Eq. (61) are negligible. By applying the relation $\xi^{2}=l /\left(T-T_{\mathrm{AFQ}}\right)$, we obtain

$$
\begin{aligned}
\chi_{66}^{\mathrm{TO}} & \approx X \xi^{2} T \\
& \approx b \frac{T}{T-T_{\mathrm{AFQ}}},
\end{aligned}
$$

where $b=X l$. The first line in Eq. (62) coincides with Eq. (49) since $\sum_{\boldsymbol{q}}\left\{\chi_{x z}^{Q}(\boldsymbol{q}, 0)\right\}^{2}=c^{2} \xi^{2} / 4 \pi$. In the opposite case (ii) $\omega_{0} \gg T$, the term $T$ in the curly brackets in Eq. (61) is negligible. Taking the relations $\psi(x) \approx \log (x)$ for $x \gg 1$ and $\omega_{0} \xi^{2} \propto \xi^{0}$ into account, we obtain

$$
\begin{aligned}
\chi_{66}^{\mathrm{TO}} & \approx X \xi^{2} \omega_{0} \log \left(2+\pi^{2} \xi^{2}\right) \\
& \approx b_{66}^{\prime} \log \left(\frac{\pi^{2} l}{T-T_{\mathrm{AFQ}}}\right)
\end{aligned}
$$

where $b^{\prime}=X \xi^{2} \omega_{0}$. Therefore, in the case (ii) $T_{\mathrm{AFQ}}<0$ and $\omega_{0} \gg T, \tilde{\chi}_{66}$ in Eq. (51) is given by replacing $\chi_{66}^{\mathrm{TO}}$ with $b_{66}^{\prime} \log \left(\pi^{2} l /\left(T-T_{\mathrm{AFQ}}\right)\right)$ in Eq. (52). In this case, the temperature dependence of $\chi_{66}^{\mathrm{TO}}$ is much moderate. In Sec. VI B, we will discuss the temperature dependence of $C_{66}$ based on Eq. (61).

In the above derivation, we have neglected the effect of mass-enhancement factor brought by the third point vertex. If we take this effect into account, both Eqs. (62) and (63) are multiplied by the factor $\left(m^{*} / m\right)^{2}=2^{2} \sim 3^{2}$, as we will discuss in Sec. VIB.

\section{DISCUSSIONS}

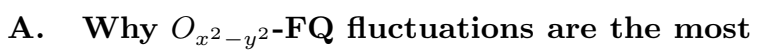 divergent for $T_{\mathrm{AFQ}}>0$ ?}

In this paper, we have studied the development of quadrupole susceptibilities in iron pnictides based on the 
RPA and beyond the RPA. The main fluctuations in the present study is the $O_{x z / y z}$-AFQ fluctuations, $\chi_{x z / y z}^{Q}(\boldsymbol{Q})$, which are produced by Fe-ion in-plane optical phonons. The acoustic phonons $\boldsymbol{q} \sim \boldsymbol{Q}$ with finite energy also assist in producing the AFQ fluctuations; see Eq. (41). We also find that the $O_{x^{2}-y^{2}}-\mathrm{FQ}$ fluctuations, $\chi_{66}$, are induced by the "two-orbiton process" described by the AL-type diagram in Fig. 8 (c). Here, the anharmonic three-phonon coupling is produced by the three-point vertex in Eq. (45). The two-orbiton process is important in iron pnictides because of the two-dimensionality.

We discuss on the total susceptibility by including the electron-acoustic phonon interaction, $\tilde{\chi}_{\Gamma}^{Q}(\boldsymbol{q})$, by taking the two-orbiton process into account. For $\Gamma=x z$ and $x^{2}-y^{2}$,

$$
\begin{aligned}
\tilde{\chi}_{x z}^{Q}(\boldsymbol{Q}) & =\frac{\chi_{x z}^{0}(\boldsymbol{Q})}{1-\left(g+g_{44}\right) \chi_{x z}^{0}(\boldsymbol{Q})}, \\
\tilde{\chi}_{x^{2}-y^{2}}(\mathbf{0}) & =\frac{\chi_{66}^{0}(\mathbf{0})+\chi_{66}^{\mathrm{TO}}}{1-g_{66}\left(\chi_{66}^{0}(\mathbf{0})+\chi_{66}^{\mathrm{TO}}\right)},
\end{aligned}
$$

where $\chi_{66}^{\mathrm{TO}}$ is proportional to the square of the AFQ correlation length $\xi^{2} \propto \tilde{\chi}_{x z}^{Q}(\boldsymbol{Q})$. When $g_{66}=0$, therefore, $\tilde{\chi}_{x z}^{Q}(\boldsymbol{Q})$ and $\tilde{\chi}_{x^{2}-y^{2}}^{Q}(\mathbf{0})$ diverges at the same time in proportion to $\xi^{2}$. As $g_{66}$ increases from zero, only $\tilde{\chi}_{x^{2}-y^{2}}^{Q}(\mathbf{0})$ is enhanced because of the absence of the two-orbiton process; $A_{\Gamma}(x z, x z ; \boldsymbol{q}), A_{\Gamma}(y z, y z ; \boldsymbol{q}) \ll 1$ for $\Gamma=x z$ or $y z$ as shown in TABLE I. For this reason, the relation $T_{S}>T_{\mathrm{AFQ}}$ is universally satisfied even if a fully selfconsistent calculation is performed.

As results, both the AFQ fluctuations (=origin of high$T_{\mathrm{c}}$ ) and the FQ fluctuations (=origin of shear modulus softening) develop at the same time, and latter fluctuations overcome the former near the $T_{S}$. The orthorhombic phase transition in under-doped compounds is brought by the divergence of the two-orbiton process $\tilde{\chi}_{66}$.

\section{B. Softening of $C_{66}$ : comparison between theory and experiment}

Is this subsection, we discuss the softening of $C_{66}$ in under- and over-doped iron pnictides based on the results in Sec. V. For this purpose, we first estimate the magnitude of the three-point vertex $A_{x^{2}-y^{2}}(x z, x z, \boldsymbol{Q})$ based on the Ward identity given in Eq. (50). Since $\bar{\chi}_{x z, x z}^{Q}(\boldsymbol{Q}, 0 ; 0) \sim(2 g)^{-1},\left|A_{x^{2}-y^{2}}\right| \sim(2 g)^{-1} / \delta E$, where $\delta E$ is the bandwidth of the $x z / y z$ band. Since $2 g \sim 0.5 \mathrm{eV}$ and $\delta E \sim 2 \mathrm{eV}$ according to the band calculations ${ }^{2}$, and considering the effect of band renormalization due to the mass-enhancement factor $m^{*} / m(=2 \sim 3)^{58}$, we expect $\left|A_{x^{2}-y^{2}}\right| \sim 1\left(m^{*} / m\right)\left[\mathrm{eV}^{-2}\right]$. This rough estimation is consistent with the numerical result in Fig. 9 (a).

Now, we discus the under-doped case with $T_{\mathrm{AFQ}}>0$. In this case, $\chi_{66}^{\mathrm{TO}} / T \approx X \xi^{2}$ shown in Eq. (62). Using the relations $c \sim 12$ and $6 \xi^{2} \sim\left(1-\alpha_{c}\right)^{-1}$ as discussed in the caption of Fig 4 , we obtain $X \sim 0.7$ and $\chi_{66}^{\mathrm{TO}} \sim$ $0.12\left(m^{*} / m\right)^{2}\left(1-\alpha_{c}\right)^{-1}$. This estimation is consistent with the numerical result Fig. 9 (b) if we put $\left(m^{*} / m\right)=$ 1.

We also discuss the optimum or over-doped systems without structure transition, in which the relation $\omega_{0} \gg$ $T$ is satisfied. In this case, $\chi_{66}^{\mathrm{TO}} \sim b^{\prime} \log \left(\pi^{2} l /\left(T-T_{\mathrm{AFQ}}\right)\right)$. Since the temperature dependence of $\chi_{66}^{\mathrm{TO}}$ is moderate, $\chi_{66}^{\mathrm{TO}}$ would be comparable or smaller than $a_{66}$. Therefore, in over-doped systems, the softening in $C_{66}$ would be much moderate, showing a deviation from the CurieWeiss type form in $\mathrm{Eq} .(55)$.

Now, we analyze the temperature dependence of $\chi_{66}^{\mathrm{TO}}$ and $C_{66} / C_{66,0}$ by using Eq. (61). We can fix the prefactor $(2 g)^{4} c^{2} \xi^{2}\left\{A_{x^{2}-y^{2}}\right\}^{2} / 4 \pi \equiv X \xi^{2}$ in front of Eq. (61) based on the relation $\chi_{66}^{\mathrm{TO}} / T=X \xi^{2}$ for $\omega_{0} \ll T$ : We obtain $X \sim 0.6$ according to Fig. 9 (b). Hereafter, we put $X=5.4$ by multiplying the square of the mass-enhancement factor, $\left(m^{*} / m\right)^{2} \sim 9$. We also put $\omega_{0}=l^{\prime}\left(T-T_{\mathrm{AFQ}}\right)$ with $l^{\prime}=2$, and $\xi^{2}=l\left(T-T_{\mathrm{AFQ}}\right)^{-1}$ with $l=0.086[\mathrm{eV}]$, which means that $\xi \sim 2$ when $T-T_{\mathrm{AFQ}}=250 \mathrm{~K}$. Using the obtained $\chi_{66}^{\mathrm{TO}}$, we plot $C_{66} / C_{66,0}$ in Fig. 10 (b) based on Eqs. (51) and (52). Here, we set $g_{66}=0.17 \mathrm{eV}$ and $a_{66}=2.5 \mathrm{eV}^{-1}$. In the case of $T_{\mathrm{AFQ}}=100 \mathrm{~K}$, we obtain $E_{\mathrm{JT}} \approx 27 \mathrm{~K}$. In the FLEX approximation $^{11}, T_{\mathrm{AFQ}}$ changes from positive to negative by carrier doping, while other parameters $\left(X, l, l^{\prime}\right.$ and $\left.a_{66}\right)$ are insensitive to the doping. Similarly to Fig. 10 (b), we can fit the recent experimental data by Yoshizawa et $a l .{ }^{32}$ for $\mathrm{Ba}\left(\mathrm{Fe}_{1-x} \mathrm{Co}_{x}\right)_{2} \mathrm{As}_{2}$ with $x=0 \sim 0.1$, by choosing $T_{\mathrm{AFQ}}$ while other parameters $\left(X, l, l^{\prime}\right.$ and $\left.a_{66}\right)$ are fixed. This fact is a strong evidence for the success of orbital fluctuation theory in iron pnictide superconductors.

In the present paper, we consider that the origin of high- $T_{\mathrm{c}}$ is the AFQ fluctuations. On the other hand, Yanagi et $a l^{43}$ claimed that high- $T_{\mathrm{c}}$ originates from the FQ fluctuations that give the softening in $C_{66}$ : In the latter mechanism, a rough estimation of $T_{\mathrm{c}}$ is given as

$$
T_{\mathrm{c}} \sim \omega_{c} \exp (-(1+\beta \lambda) / \beta \lambda)
$$

where $\omega_{c}$ is the phonon energy relevant for the orbital fluctuations, which is just $\sim 10 \mathrm{~K}$ for $|\boldsymbol{q}| \sim 0.1 \pi$. $\beta \equiv$ $1+g_{66} \tilde{\chi}_{66}=C_{66,0} / C_{66}$ is the enhancement factor due to FQ fluctuations ${ }^{44}$. However, $C_{66,0} / C_{66}$ observed in optimally-doped $\mathrm{Ba}(\mathrm{Fe}, \mathrm{Co})_{2} \mathrm{As}_{2}$ is just $\sim 1.2^{30,32}$ : Apparently, such small enhancement cannot reproduce high$T_{\mathrm{c}}$ superconductivity in iron-pnictides.

In the present study, in contrast, weak softening in optimally-doped sample is ascribed to the change in the scaling of $\chi_{66}^{\mathrm{TO}}$, not to the weakness of AFQ fluctuations. In fact, the softening is moderate in the case of $T_{\mathrm{AFQ}}=-10 \mathrm{~K}$ in Fig. 10, while the AFQ correlation $\xi^{2} \approx 1000 /(T[\mathrm{~K}]+10)$ is enough to cause the superconductivity at $T_{\mathrm{c}} \sim 30 \mathrm{~K}$. Therefore, moderate softening and high- $T_{\mathrm{c}}$ are compatible in the present study. 

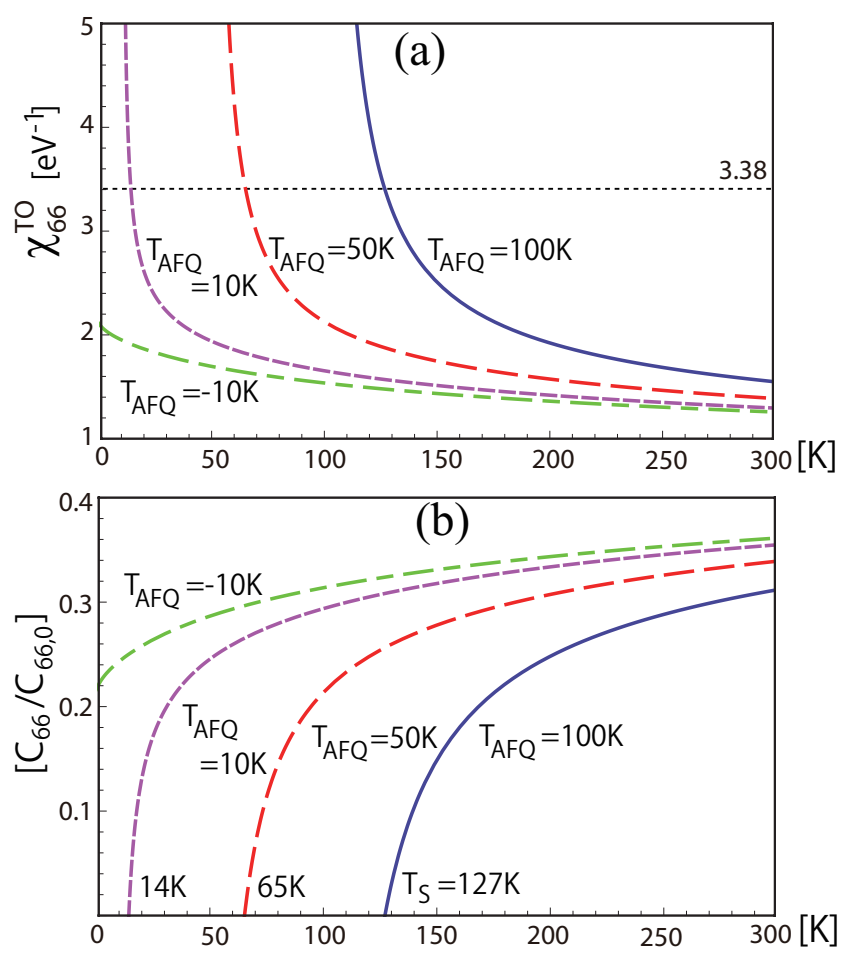

FIG. 10: (Color online) (a) $\chi_{66}^{\mathrm{TO}}$ for $T_{\mathrm{AFQ}}=100 \mathrm{~K}, 50 \mathrm{~K}, 10 \mathrm{~K}$ and $-10 \mathrm{~K}$. given by Eq. (61). (b) $C_{66} / C_{66,0}$ for $T_{\mathrm{AFQ}}=$ $100 \mathrm{~K}, 50 \mathrm{~K}, 10 \mathrm{~K}$ and $-10 \mathrm{~K}$. given by Eqs. (51) and (52). We put $X=5.7, l=0.086, l^{\prime}=2, g_{66}=0.17$, and $a_{66}=2.5$. $C_{66}=0$ is realized when $\chi_{66}^{\mathrm{TO}}=g_{66}^{-1}-a_{66}$, which is 3.38 in the present parameters. Using these same parameters, we can fit the recent experimental data by Yoshizawa et al. ${ }^{32}$ for $\mathrm{Ba}\left(\mathrm{Fe}_{1-x} \mathrm{Co}_{x}\right)_{2} \mathrm{As}_{2}$ for $x=0 \sim 0.1$, just by changing $T_{\mathrm{AFQ}}$.

\section{Quadrupole-ordered state in under-doped compounds}

Here, we consider the orbital or quadrupole ordered state in under-doped compounds. In the mean-field approximation for the multiorbital Hubbard model for iron pnictides ${ }^{59,60}$, stripe-type SDW order occurs for $U>U_{\mathrm{c}}$, and weak orbital polarization $\left(n_{x z} \neq n_{y z}\right)$ is induced as the secondary order when the magnetization is large. However, in real materials, orthorhombic transition occurs in the paramagnetic state, and the SDW-order is induced in the orthorhombic phase. To solve this problem, we studied the multiorbital $\mathrm{HH}$ model beyond the mean-field theory, and found that the FQ order occurs in the paramagnetic state due to the two-orbiton process. Fortunately, this FQ order does produce the experimentally observed SDW order, as we will explain in the next subsection.

As discussed in Sec. VB, the divergence of $\tilde{\chi}_{66}$, which is the total FQ susceptibility given by both optical and acoustic phonons, causes the orthorhombic structure transition when $C_{66}=0$. The $O_{x^{2}-y^{2}}-\mathrm{FQ}$ order is realized in the orthorhombic phase. The schematic quadrupole order is shown in Fig. 11 (a). Since $O_{x^{2}-y^{2}} \approx$ $n_{2}-n_{3}$ according to Eq. (7), the order parameter $O_{x^{2}-y^{2}}>0(<0)$ corresponds the orbital polarized state with $n_{x z}>n_{y z}\left(n_{x z}<n_{y z}\right)$. Figure 11 (b) shows the $\mathrm{AFQ}$ order brought by the divergence of $\chi_{x z}^{Q}(\boldsymbol{Q})$. Although the FQ order in (a) would occur earlier, we expect the AFQ order in (b) would coexist with the FQ order when the structure transition is the weak first order. In fact, the reconstruction of the FSs above $T_{\mathrm{N}}$ in detwinned $\mathrm{Ba}\left(\mathrm{Fe}_{1-x} \mathrm{Co}_{x}\right)_{2} \mathrm{As}_{2}{ }^{25}$ would indicate the presence of the AFQ order ${ }^{61}$.

(a)
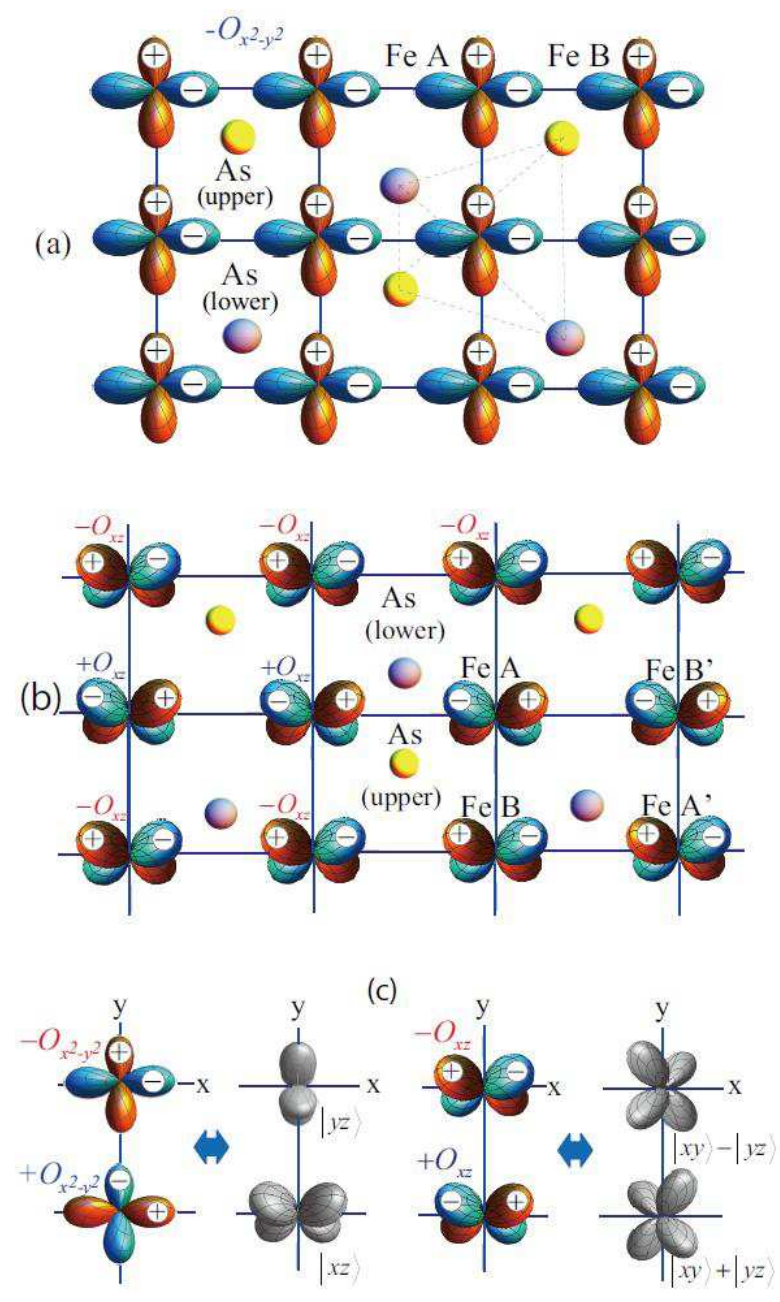

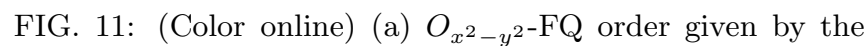
divergence of $C_{66}^{-1}$. Be careful not to confuse $\hat{O}_{x^{2}-y^{2}}$ with the $x^{2}-y^{2}$-orbital operator. (b) $O_{x z}$-AFQ order brought by the divergence of $\chi_{x z}^{Q}(\boldsymbol{Q})$. (c) The correspondence between

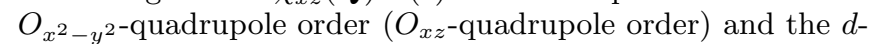
orbital with larger occupation number.

In Fig. 11 (c), we show the correspondence between the quadrupole order and the $d$-wavefunction with larger electron occupancy. In the $O_{x^{2}-y^{2}}$-type quadrupole order, the electrons mainly occupy the state $|x z\rangle$ for $O_{x^{2}-y^{2}}>0$, or the state $|y z\rangle$ for $O_{x^{2}-y^{2}}<0$. In the $O_{x z}$-type quadrupole order, the electrons mainly occupy the state $|x y\rangle+|x z\rangle$ for $O_{x z}>0\left(|x y\rangle-|x z\rangle\right.$ for $\left.O_{x z}<0\right)$. 
Finally, we make comparison between the present study and the previous work based on the RPA ${ }^{43}$, which claims that the divergence of Eq. (51) is caused by large $g_{66} a_{66} \lesssim 1$ while neglecting $\chi_{66}^{\mathrm{TO}}$. However, the obtained

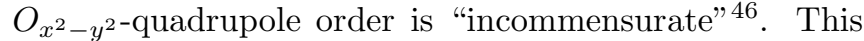
result highlights the importance of the two-orbiton process $\chi_{66}^{\mathrm{TO}}$ in order to produce the " $\boldsymbol{q}=0$ " orthorhombic structure transition.

\section{Stripe magnetic order produced by $O_{x^{2}-y^{2}}-\mathbf{F Q}$ order}

In under-doped iron pnictides, the collinear-SDW order is induced in the orthorhombic phase at $T_{\mathrm{N}}$, which is slightly lower temperature than $T_{S}$. Various explanations for the origin of this SDW transition had been proposed previously. From a strong-coupling scheme, square-lattice Heisenberg model with in-plane anisotropy, $J_{1 a^{-}} J_{1 b^{-}} J_{2}$ model, had been studied ${ }^{26}$. According to the neutron scattering on $\mathrm{CaFe}_{2} \mathrm{As}_{2}{ }^{34}$, the high-energy spinwave dispersion indicates the relation $J_{1 a} \gg J_{1 b}$ in the orthorhombic phase. In this case, experimentally observed staggered spin order along the $x$-axis ( $a$-axis) is expected to be realized. However, such strong in-plane anisotropy $J_{1 a} \gg J_{1 b}$ is surprising, considering the small orthorhombicity $(a-b) /(a+b) \sim 0.003$. These fact would indicate the existence of orbital or quadrupole order in the orthorhombic phase.

In this subsection, we study the origin of SDW state based on the weak-coupling approach. Hereafter, we assume that $x$-axis corresponds to $a$-axis (longer lattice constant) in the orthorhombic phase. In the previous subsection, we explained that the two-orbital process induces the $O_{x^{2}-y^{2}}-\mathrm{FQ}$ order in Fig. 11. Note that $O_{x^{2}-y^{2}} \approx n_{2}-n_{3}$ according to Eq. (7). The corresponding mean-field is given as

$$
H^{\prime}=\Delta E \sum_{i}(|2\rangle\langle 2|-| 3\rangle\langle 3|)_{i},
$$

which raises (lowers) the energy-level of orbital 2 (3) by $\Delta E$. In a similar model, the change in the DOS and FSs by the orthorhombic potential $\Delta E$ was studied by Chen et al..$^{62}$. Here, we study the change in the spin susceptibility by $\Delta E$ using the RPA.

We calculate the total spin susceptibility $\chi^{s}(\boldsymbol{q}, 0)=$ $\sum_{l, m} \chi_{l, l ; m, m}^{s}(\boldsymbol{q}, 0)$ for $U=1.1$ and $g=0$. Figure 12 (a) shows the obtained $\chi^{s}(\boldsymbol{q}, 0)$ for $\Delta E=0$; the corresponding spin Stoner factor is $\alpha_{S}=0.87$. When $\Delta E$ is finite, the four-hold symmetry in $\chi^{s}(\boldsymbol{q}, 0)$ disappears quickly. Figure $12(\mathrm{~b})$ shows the change in the spin susceptibility, $\chi^{s}(\boldsymbol{q} ; \Delta E)-\chi^{s}(\boldsymbol{q} ; 0)$, induced by $\Delta E=+0.04$. We see that $\chi^{s}(\boldsymbol{q}, 0)$ increases by +6.5 at $\boldsymbol{q}=(0, \pi)$ while decreases by -4.0 at $\boldsymbol{q}=(\pi, 0)$. Therefore, magnetic frustration is resolved and stripe-SDW order can be induced by small $\Delta E$.

Figure 12 (c) shows the $\Delta E-U_{c}$ phase diagram given by the RPA, that is, by the mean-field-approximation.

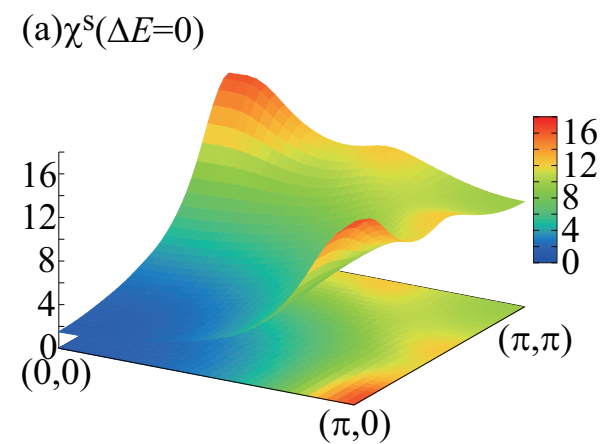

(b) $\chi^{\mathrm{S}}(\Delta E=+0.04)-\chi^{\mathrm{S}}(\Delta E=0)$
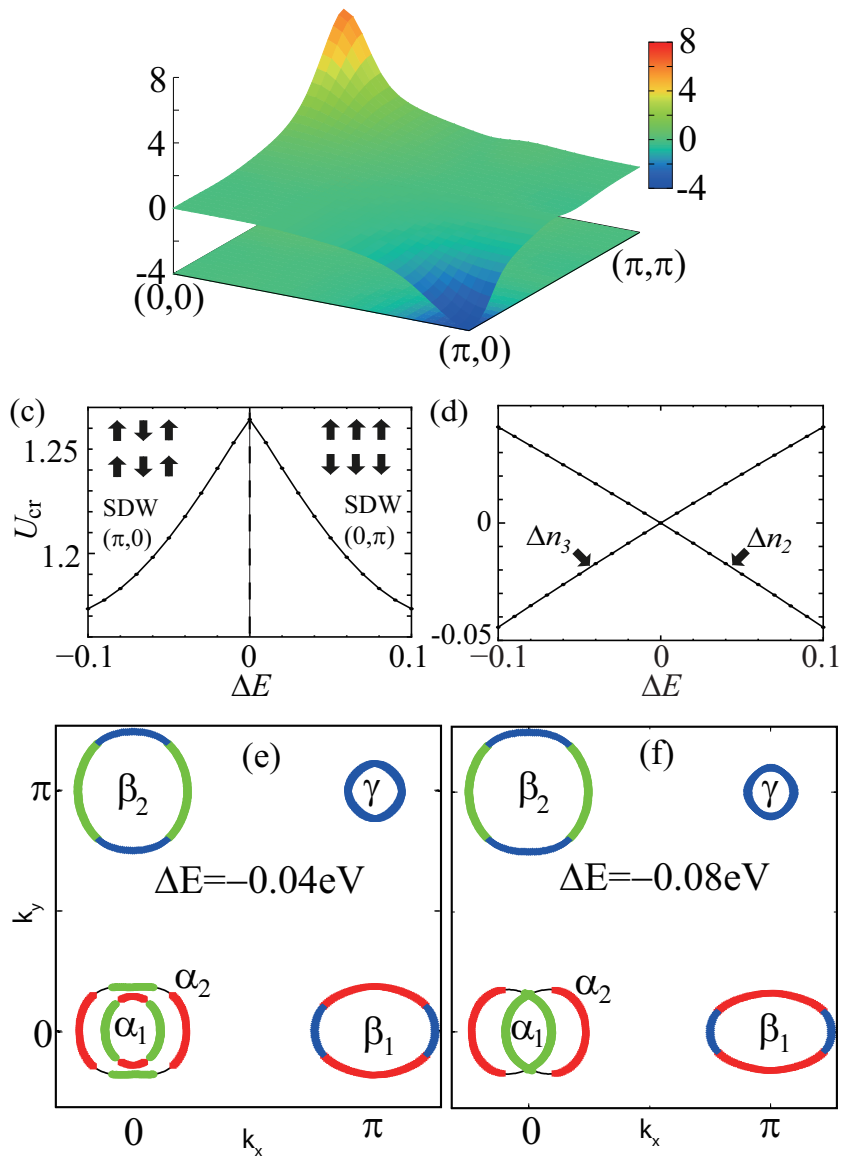

FIG. 12: (Color online) (a) $\chi^{s}(\boldsymbol{q} ; \Delta E)$ for $\Delta E=0$. Used model parameters are $n=6.05, U=1.1, g=0$, and $T=0.05$ (b) $\chi^{s}(\boldsymbol{q} ; \Delta E)-\chi^{s}(\boldsymbol{q} ; 0)$ for $\Delta E=0.04$. Model parameters are the same as those in (a). (c) $U_{c}$ as function of $\Delta E$ given by the RPA for $m^{*} / m=1$. (d) $\Delta n_{2}$ and $\Delta n_{3}$ as function of $\Delta E$ for $m^{*} / m=1$. Note that $\Delta n \equiv \Delta n_{2}-\Delta n_{3}$. (e) FSs for $\Delta E=-0.04$ for $n=6.05$. We use the same color coding as in Fig. 2. (f) FSs for $\Delta E=-0.08$ for $n=6.05$. In both cases, the best nesting vector is $\boldsymbol{q}=(\pi, 0)$.

It is noteworthy that $U_{c}$ quickly decreases in proportion to $|\Delta E|$, because of the degeneracy of orbital 2 and 3 . When $U \lesssim U_{\mathrm{c}}$, the experimental SDW order with momentum $\boldsymbol{q}=(\pi, 0)$ is realized by the negative potential $\Delta E$ that corresponds to $n_{2}>n_{3}$. Figure 12 (d) gives the 
relation between $\Delta n=\Delta n_{2}-\Delta n_{3}$ and $\Delta E$ : If we take the band-renormalization effect into account, we obtain the relation $\Delta n=-0.85\left(\mathrm{~m}^{*} / \mathrm{m}\right) \Delta E$. According to (c) and (d), we obtain the reduction in $U_{c}$ due to the FQ order is

$$
\Delta U_{c}=-1.4|\Delta n|=-1.2\left(m^{*} / m\right)|\Delta E| .
$$

Therefore, only few percent $\Delta n$ can induce large change in $U_{\mathrm{c}}$ that is linear in $|\Delta n|$. According to recent ARPES measurement in detwinned $\mathrm{BaFe}_{2} \mathrm{As}_{2}{ }^{25}, \Delta E \sim-0.03 \mathrm{eV}$ in the orthorhombic phase, which corresponds to $\Delta n=$ $+0.026\left(\mathrm{~m}^{*} / \mathrm{m}\right)$ and $\Delta U_{c}=-0.036\left(\mathrm{~m}^{*} / \mathrm{m}\right)$ in the present five-orbital model. In this case, the realized SDW order is $\boldsymbol{q}=(\pi, 0)$, which is consistent with famous strip-type SDW state in mother compounds ${ }^{22}$.

We can show that the SDW temperature $T_{\mathrm{N}}$ also increases linearly in $|\Delta n|$ based on the Landau theory. The free energy in the present problem would be given as

$$
F(\Delta n)=F(0)+c \Delta n\left(m_{(\pi, 0)}^{2}-m_{(0, \pi)}^{2}\right),
$$

where $m_{\boldsymbol{Q}}$ is the $\mathrm{AF}$ order with momentum $\boldsymbol{Q}$, and $F(0)=a \cdot\left(T-T_{\mathrm{N}}^{0}\right)\left(m_{(\pi, 0)}^{2}+m_{(0, \pi)}^{2}\right)+b\left(m_{(\pi, 0)}^{4}+m_{(0, \pi)}^{4}\right) / 2+$ $\cdots$ with $a, b>0$. Then, we obtain $T_{\mathrm{N}}=T_{\mathrm{N}}^{0}+|c \Delta n| / a$. The present study shows that $c<0$, which seems consistent with the numerical result in Ref. 60 .

Now, we consider the reason why SDW order is produced by $\Delta E$ : Figure 12 (e) and (f) shows the change in the the FS structure with $\Delta E$. We can recognize that the intra-orbital (orbital 3) nesting between FS $\alpha_{2}$ and FS $\beta_{1}$ becomes better, compared to the case of $\Delta E=0$ in Fig. 2 (a). Therefore, the origin of the "FQ-order-induced stripe-SDW" is the "anisotropy in the intra-orbital nesting" caused by small $|\Delta E| \sim 0.03 \mathrm{eV}$, which corresponds to a small orbital polarization $|\Delta n| \sim 0.026\left(\mathrm{~m}^{*} / \mathrm{m}\right)$. This result is consistent with the very small orthorhombicity $(a-b) /(a+b) \lesssim 0.003$ in the orthorhombic state $^{22}$. In the strong-coupling description, the origin of the stripe-SDW state is the in-plane anisotropy in the exchange interaction $\left(J_{1 a} \neq J_{1 b}\right)^{26,34}$ brought by twoorbiton process.

If we go beyond the RPA, the SDW state will be further stabilized by the reduction in the quasiparticle damping $\gamma$ when the FQ-order is established ${ }^{11}$ : In fact, in the FLEX approximation ${ }^{11}, \chi^{s}(\boldsymbol{q})$ is suppressed by $\gamma$ due to strong orbital fluctuations in the normal state. Since the orbital fluctuations is suppressed when the AFQ-order sets in, the resultant increment in $\chi^{s}(\boldsymbol{q})$ would stabilize the SDW phase.

\section{E. Summary}

In the present paper, we have studied the realistic fiveorbital HH model for iron pnictides. In the RPA, only the $O_{x z}$-AFQ fluctuations develop as shown previously ${ }^{9}$, and therefore the softening of shear moduli $\left(C_{66}, C_{44}\right.$ and $C_{E}$ ) cannot be reproduced. In the present study beyond

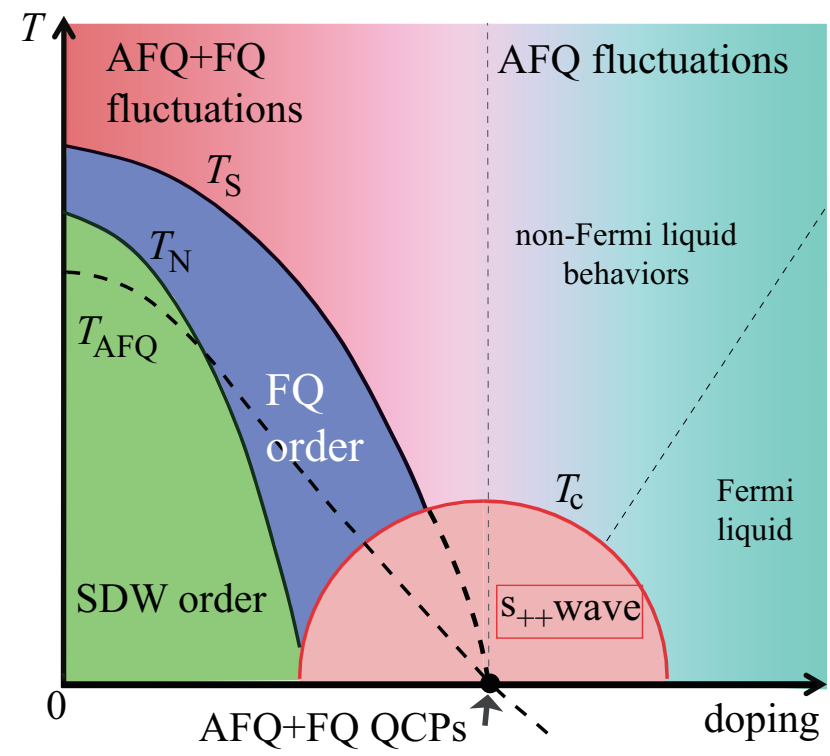

FIG. 13: (Color online) The phase-diagram for iron-pnictide superconductors obtained by the present orbital fluctuation theory. $T_{S}$ is the orthorhombic transition temperature $(=\mathrm{FQ}$ order temperature), and $T_{\mathrm{N}}$ is SDW transition temperature. The fact that two QCPs at $T_{S}=0$ and $T_{\mathrm{AFQ}}=0$ almost coincide means that novel "multi orbital QCPs" are realized in iron pnictides. The left-hand (right-hand) side of the vertical dotted line corresponds to $T_{\mathrm{AFQ}}>0\left(T_{\mathrm{AFQ}}<0\right)$, in which the two-orbiton process is relevant (irrelevant). At $T_{\mathrm{AFQ}}$, the AFQ-order does not occur since it is prevented by the FQorder at $T_{S}$.

the RPA, we revealed that both $O_{x z}-\mathrm{AFQ}$ fluctuations and $O_{x^{2}-y^{2}}$ FQ fluctuations develop at the same time. The former and the latter fluctuations are the origins of the $s_{++}$-wave superconductivity and the orthorhombic structure transition, respectively. The commensurate FQ fluctuations are brought by the two-orbiton process in Fig. 8 (c) that is dropped in the RPA. [In the meanfield theory, the orbital order due to large $g_{66}$ is always "incommensurate" ${ }^{46}$.] Fluctuation-induced softening occurs only in $C_{66}$ out of three shear moduli because of the orbital selection rule for the three-point vertex. The origin of softening would be interpreted as "virtual anharmonicity of lattice vibrations" that is induced by AFQ fluctuations; see Fig.8 (b). Possible quadrupole orders in the ordered state are show in Fig. 11. Using the two-orbiton term in Eq. (61), we can fit the recent experimental data of $C_{66}$ in $\mathrm{Ba}\left(\mathrm{Fe}_{1-x} \mathrm{Co}_{x}\right)_{2} \mathrm{As}_{2}{ }^{32}$ for wide range of doping, only by choosing $T_{\mathrm{AFQ}}$ while other parameters are fixed. This fact is a strong evidence for the success of orbital fluctuation theory in iron pnictide superconductors.

In addition, we should stress that the stripe-type antiferro-magnetic state is realized in the orbital-ordered state, since the small orbital polarization $(\Delta n \lesssim 0.05)$ can cause large in-plane anisotropy in the exchange interaction $\left(J_{1 a} \neq J_{1 b}\right)$. Thus, the present study presents 
a microscopic justification for the anisotropic Heisenberg model description for the SDW state ${ }^{26,34}$.

In Fig. 13, we summarize the phase-diagram of ironpnictides given by the present orbital fluctuation theory beyond the RPA. We stress that $T_{\mathrm{AFQ}}$, which is determined experimentally from $C_{66}$, is positive in the under-doped case $\left(T_{S}>0\right)$ while it is negative in the over-doped case, as recognized from Eq. (54) obtained in the classical approximation. Especially, $T_{S} \approx 0$ for $T_{\mathrm{AFQ}}=0$, consistently with experiments ${ }^{31,33}$. This result indicats that QCPs for $\mathrm{AFQ}$ and $\mathrm{FQ}$ orders almost coincide at the endpoint of the orthorhombic phase. The emergence of "multi orbital QCPs" is favorable to the orbital-fluctuation-mediated $s_{++}$-wave SC state ${ }^{9-11}$. In fact, $T_{\mathrm{AFQ}}$ is derived from experimentally observed $C_{66}$ as follows: In under-doped systems with $T_{S}>0, T_{\mathrm{AFQ}}$ is given the Weiss temperature of $C_{66} \propto\left(T-T_{S}\right) /(T-\theta)$, and $T_{\mathrm{AFQ}}=\theta$ is indeed positive experimentally. In overdoped systems, both $T_{S}$ and $\theta$ are negative $\left(T_{S}>\theta\right)$, and $C_{66}$ starts to deviate from the Curie-Weiss behavior. These experimental results are the strong evedence for the realization of the two-orbiton process $\left(\chi_{66}^{\mathrm{TO}} \sim\right.$ $\left.T /\left(T-T_{\mathrm{AFQ}}\right)\right)$ in iron-pnictides. In contrast, in the cooperative Jahn-Teller scenario due to large $g_{66}$ by Yanagi et $a l .{ }^{43}$, the parameter $\theta$ is always negative; see Appendix A in detail. Finally, we note that the two-orbiton term $\chi_{66}^{\mathrm{TO}}$ in the present study is very similar to the bare nematic susceptibility $\chi_{0, \text { nem }}$ in Ref. ${ }^{30}$, which is the two-magnon term on different sublattices in our terminology.

In summary, the present study can explain the superconductivity, orthorhombic transition, and softening of $C_{66}$ due to FQ and AFQ quantum-criticalities. The stripe-SDW order is naturally produced by the "orthorhombicity" of the FQ order. These results are strong evidence for the realization of the orbital-fluctuationmediated $s_{++}$-wave superconductivity in iron pnictides. Finally, we stress that the present study enables us to derive the important parameters in the orbital fluctuation model in Eqs. (46)-(48) from the experimental data of shear modulus.

\section{Acknowledgments}

We are grateful to M. Yoshizawa for valuable discussions on his interesting experimental results. We also thank D.S. Hirashima, M. Sato, Y. Matsuda, T. Shibauchi, and R.M. Fernandes for valuable comments and discussions. This study has been supported by Grants-in-Aid for Scientific Research from MEXT of Japan, and by JST, TRIP. Numerical calculations were performed using the facilities of the supercomputer center, Institute for Molecular Science.

\section{Appendix A: Structure transitions in itinerant electron systems: Quadrupole-quadrupole interaction V.S. Cooperative Jahn-Teller effect}

In this paper, we studied the structure transition due to $O_{x^{2}-y^{2}}-\mathrm{FQ}$ order in iron-pnictides. The ferro quadrupole interaction originates from the two-orbiton process with respect to $O_{x z}$-AFQ fluctuations, which are induced by optical phonons. The $O_{x^{2}-y^{2}}$-FQ fluctuations give the softening of the elastic constant $C_{66}$.

In this section, we present a general theory for the structure transitions in itenerant metals, and consider the uniqueness of iron pnictides in the next step. The structure transition due to ferro-quadrupole order is classified as the "cooperative Jahn-Teller type" or "quadrupolequadrupole interaction type" 52 . The elastic constant $C_{\phi}$ is given by Eqs. (39) and (40), where $\chi_{\phi}$ is the quadrupole susceptibility at $\boldsymbol{q}=0$ without acoustic phonons. Now, we introduce the quadrupole-quadrupole interaction $g$ like in Eq. (10), the origin of which is the optical phonons or Coulomb interaction. In the RPA, $\chi_{\phi}=\chi_{\phi}^{0} /\left(1-g \chi_{\phi}^{0}\right)$, where $\chi_{\phi}^{0}$ is the bare quadrupole susceptibility. Then, $C_{\phi}$ is given as

$$
\frac{C_{\phi}}{C_{\phi, 0}}=\frac{1-\left(g_{\mathrm{ac}}+g\right) \chi_{\phi}^{0}}{1-g \chi_{\phi}^{0}}
$$

In rare-earth metals in which $f$-electrons are localized, $\chi_{\phi}^{0}$ is proportional to $1 / T^{51,52}$. However, this replacement is inappropriate in itinerant metals. In nearly ferroquadrupole itinerant metals, $\left|1-\left(g_{\mathrm{ac}}+g\right) \chi_{\phi}^{0}\right| \ll 1$ at zero temperature.

Here, we consider the temperature dependence of $C_{\phi}$ beyond the RPA. In the FLEX approximation ${ }^{11}$ or SCR theory ${ }^{56}, \chi_{\phi}^{0}$ is replaced with $\chi_{\phi}^{0}-\alpha T(\alpha>0)$ due to the thermal fluctuations, which are mainly described as the self-energy. In this case, Eq. (A1) becomes

$$
\frac{C_{\phi}}{C_{\phi, 0}} \approx \frac{T-T_{S}}{T-\theta}
$$

where $T_{S}=-\left(1-\left(g_{\mathrm{ac}}+g\right) \chi_{\phi}^{0}\right) /\left(g_{\mathrm{ac}}+g\right) \alpha$ and $\theta=$ $-\left(1-g \chi_{\phi}^{0}\right) / g \alpha$. Therefore, $C_{\phi}$ shows the Curie-Weiss behavior in the RPA by taking the thermal fluctuations into account.

First, we consider the case (i) $g_{\text {ac }} \ll g$, in which the structure transition is driven by quadrupole-quadrupole interaction $^{52}$. In this case, $E_{\mathrm{JT}} \equiv T_{S}-\theta \approx g_{\mathrm{ac}} / g^{2} \alpha$, which is much smaller than $T_{S}$. (Note that $C_{\phi}$ is not soften when $g_{\mathrm{ac}}=0$.) Then, the lattice distortion in the ordered state will be very small ( $\ll 1 \%)$, like in $\mathrm{PrRu}_{4} \mathrm{P}_{12}$. In the opposite case (ii) $g_{\text {ac }} \gg g$, the structure transition is driven by cooperative Jahn-Teller effect $^{52}$. In this case, $\theta \approx-1 / g \alpha$, which takes a large negative value since $\alpha$ is small; $\theta \sim-300 \mathrm{~K}$. Then, the lattice distortion in the ordered state should be very large ( $\gg 1 \%$ ), like manganites. In case (ii), the energy of the "ferro-orbital fluctuations" induced by the acoustic 
phonon is very low, and the $T_{\mathrm{c}}$ of the orbital-fluctuation superconductivity is still lower. Therefore, emergence of high- $T_{\mathrm{c}}$ superconductivity is unlikely in case (ii).

In the case of $C_{66}$ in iron pnictides, $O_{x^{2}-y^{2}-O_{x^{2}-y^{2}}}$ interaction $g$ due to optical phonons is absent. Moreover,

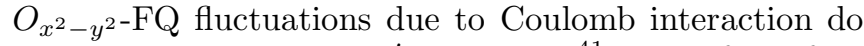
not develop even in the $U^{\prime}>U$ model $^{41}$. Therefore, if we try to explain the $C_{66}$ softening within the RPA, we have to assume the case (ii), i.e., the cooperative Jahn-Teller transitionas, as proposed by Yanagi et al. ${ }^{43}$. However, it contradicts with experiments facts, that is, $(a-b) /(a+$ $b) \lesssim 0.3 \%$ and $n_{x z}-n_{y z} \lesssim 5 \%$ in the orthorhombic phase irrespective of higher $T_{S}(\sim 100 \mathrm{~K})$, and $\theta>0$ in the under-doped pnictides. Therefore, the RPA analysis done by Yanagi et al. ${ }^{43}$ is inconsistent with experiments.

In this paper, we studied the structure transition in case (i), i.e., the quadrupole-quadrupole interaction type. The origin of quadrupole-quadrupole interaction is the "two-orbiton process", which is not taken into account in the RPA. In two-dimensional systems, the two-orbiton process gives the Curie-Weiss behavior in Eq. (A2), if allowed by the orbital selectrion rule discussed in Sec. VIB. By using reasonable sets of parameters $\left(g_{\mathrm{ac}}<g\right)$, experimentally observed Curie-Weiss behavior of $C_{66}$ in underdoped pnictides $\left(T_{S}>0\right)^{31,33}$ is well reproduced, given in Eq. (55): According to Eq. (54), $T_{\mathrm{AFQ}}(=\theta)$ is positive in under-doped pnictides, and $T_{\mathrm{AFQ}} \approx 0$ at the critical point $T_{S}=0$, consistently with experimental reports ${ }^{33}$. In over-doped pnictides, the softening of $C_{66}$ becomes moderate since the two-orbiton process gives very weak temperature dependence.

\section{Appendix B: Quadrupole susceptibilities in the ten-orbital model: effect of unfold gauge transformation}

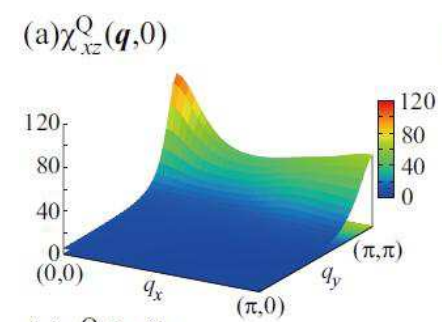

(b) $\chi_{y z}^{\mathrm{Q}}(\boldsymbol{q}, 0)$

(c) $\chi_{x y}^{\mathrm{Q}}(\boldsymbol{q}, 0)$
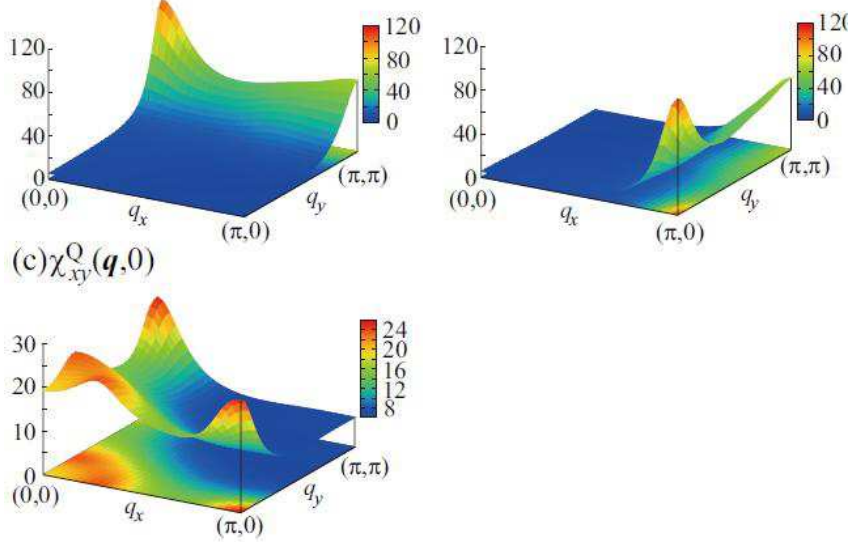

FIG. 14: (Color online) Quadrupole susceptibilities in the ten-orbital model for (a) $\chi_{x z}^{Q}(\boldsymbol{q})$, (b) $\chi_{y z}^{Q}(\boldsymbol{q})$, and (c) $\chi_{x y}^{Q}(\boldsymbol{q})$, respectively. We put $n=6.05, T=0.05$, and $\alpha_{c}=0.98$.

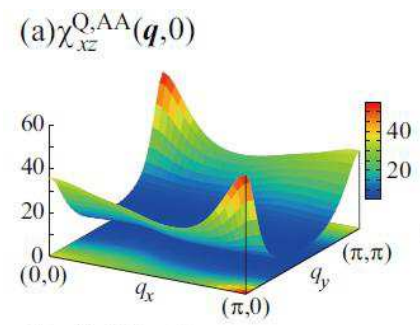

$\chi_{x z}^{\mathrm{Q}, \mathrm{AB}}(\boldsymbol{q}, 0)$

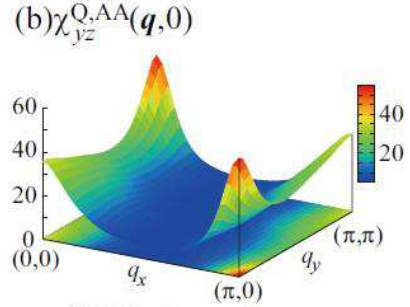

(c) $\chi_{x y}^{\mathrm{Q}, \mathrm{AA}}(\boldsymbol{q}, 0)$

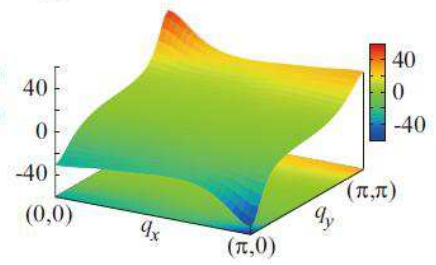

$\chi_{y z}^{\mathrm{Q}, \mathrm{AB}}(\boldsymbol{q}, 0)$
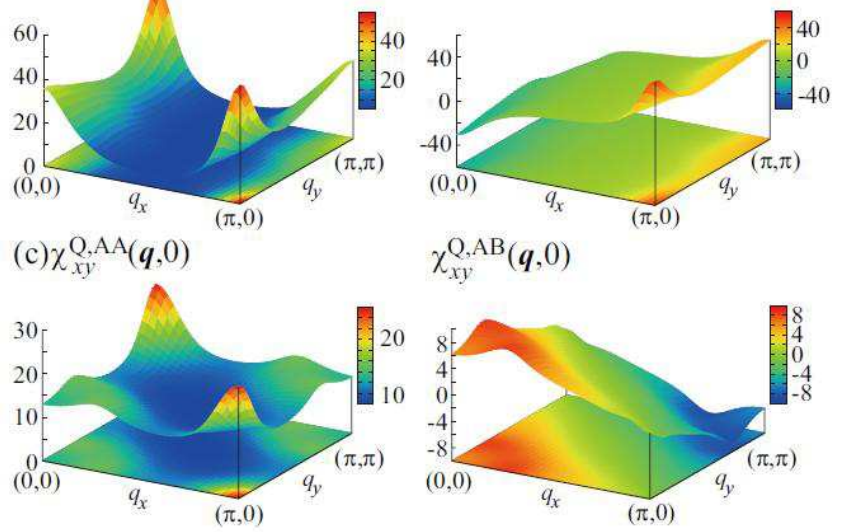

$\chi_{x y}^{\mathrm{Q}, \mathrm{AB}}(\boldsymbol{q}, 0)$

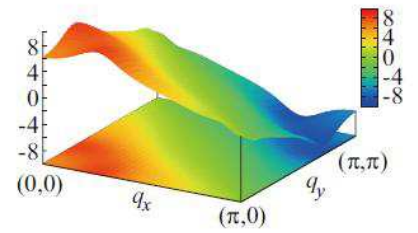

FIG. 15: (Color online) Quadrupole susceptibilities in the tenorbital model for (a) $\chi_{x z}^{Q, A A}(\boldsymbol{q})$ and $\chi_{x z}^{Q, A B}(\boldsymbol{q}),(\mathrm{b}) \chi_{y z}^{Q, A A}(\boldsymbol{q})$ and $\chi_{y z}^{Q, A B}(\boldsymbol{q})$, and $(\mathrm{c}) \chi_{x y}^{Q, A A}(\boldsymbol{q})$ and $\chi_{x y}^{Q, A B}(\boldsymbol{q})$, respectively. We put $n=6.05, T=0.05$, and $\alpha_{c}=0.98$.

In Sec. III, we have calculated $\chi_{\Gamma}^{Q}(\boldsymbol{q}, 0)$ based on the five-orbital model using the RPA, shown in Fig. 4. In this Appendix, we discuss the effect of the unfold-gauge transformation in deriving the five-orbital model on $\chi_{\Gamma}^{Q}(\boldsymbol{q}, 0)$. This gauge transition changes the signs of 2,3 -orbitals for Fe-B sites. Therefore, signs of quadrupole operators $\hat{O}_{x z / y z}^{i}$ at Fe-B sites are reversed, as recognized in Eqs. (4)-(8). For this reason, $\chi_{x z, y z}^{Q}(\boldsymbol{q}, \omega)$ is not gaugeinvariant since it contains linear terms with respect to $\hat{O}_{x z / y z}^{i}$, although the el-el interaction in Eqs. (14)-(16) is gauge invariant.

From now on, we calculate the quadrupole susceptibilities in the ten-orbital model. We describe the orbitals of Fe-A (Fe-B) ions as $1 \sim 5(6 \sim 10)$. In the RPA, the susceptibilities are given as

$$
\begin{aligned}
\chi_{\Gamma}^{Q}(q) & =\chi_{\Gamma}^{Q, A A}(q)+\chi_{\Gamma}^{Q, A B}(q), \\
\chi_{\Gamma}^{Q, A A(A B)}(q) & =\sum_{l l^{\prime}}^{A} \sum_{m m^{\prime}}^{A(B)} o_{\Gamma}^{l l^{\prime}} \chi_{l l^{\prime} m m^{\prime}}^{c}(q) o_{\Gamma}^{m m^{\prime}},
\end{aligned}
$$

for $\Gamma=x z, y z, x y$, where $o_{\Gamma}^{l m}=o_{\Gamma}^{l-5, m-5}$ for $l, m \geq 6$. The obtained quadrupole susceptibilities in Eq. (B1) is show in Fig. 14, and its diagonal and off-diagonal terms with respect to $\mathrm{A}$ and $\mathrm{B}$ are also shown in Fig. 15. In these figures, we have unfolded the susceptibilities into the single-iron BZ in order to make comparison with Fig. 4. According to Eq. (B1), $\chi_{\Gamma}^{Q}(q)$ in the ten-orbital model 
is given by $\chi_{\Gamma}^{Q, A A}(q)+\chi_{\Gamma}^{Q, A B}(q)$. We see that $\chi_{x z}^{Q}(\boldsymbol{q}, 0)$ is Fig. 14 (a) has a sharp peak at $\boldsymbol{q}=(0, \pi)$, since both $\chi_{x z}^{Q, A A}(q)$ and $\chi_{x z}^{Q, A B}(q)$ in Fig. 15 (a) have peaks at $\boldsymbol{q}=(0, \pi)$.

If we perform the unfold-gauge transformation, the sign of $\chi_{\Gamma}^{Q, A B}(q)$ is inverted for $\Gamma=x z$ and $y z$. Therefore, in the five-orbital model, $\chi_{\Gamma}^{Q}(q, 0)=\chi_{\Gamma}^{Q, A A}(q)-$
$\chi_{\Gamma}^{Q, A B}(q)$ for $\Gamma=x z$ and $y z$. For this reason, the peak in $\chi_{x z}^{Q}(\boldsymbol{q}, 0)$ move from $\boldsymbol{q}=(0, \pi)$ to $(\pi, 0)$ under the gauge transformation, consistently with the result in Fig. 4 (a). In contrast to $\chi_{x z / y z}^{Q, A B}(\boldsymbol{q}), \chi_{x y}^{Q}(\boldsymbol{q})$ is gauge invariant. For this reason, Fig. 14 (c) in the ten-orbital model coincides with Fig. 4 (c) in the five-orbital model.
1 Y. Kamihara, T. Watanabe, M. Hirano, and H. Hosono: J. Am. Chem. Soc. 130, 3296 (2008).

${ }^{2}$ K. Kuroki, S. Onari, R. Arita, H. Usui, Y. Tanaka, H. Kontani, and H. Aoki, Phys. Rev. Lett. 101, 087004 (2008).

3 I. I. Mazin, D. J. Singh, M. D. Johannes, and M. H. Du, Phys. Rev. Lett. 101, 057003 (2008).

${ }^{4}$ S. Graser, G. R. Boyd, C. Cao, H.-P. Cheng, P. J. Hirschfeld, and D. J. Scalapino, Phys. Rev. B 77, 180514(R) (2008).

5 A. V. Chubukov, D. V. Efremov, and I. Eremin, Phys. Rev. B 78, 134512 (2008).

${ }^{6}$ S. Onari and H. Kontani, Phys. Rev. Lett. 103177001 (2009).

7 A. Kawabata, S. C. Lee, T. Moyoshi, Y. Kobayashi, and M. Sato, J. Phys. Soc. Jpn. 77 (2008) Suppl. C 103704; M. Sato, Y. Kobayashi, S. C. Lee, H. Takahashi, E. Satomi, and Y. Miura, J. Phys. Soc. Jpn. 79 (2009) 014710; S. C. Lee, E. Satomi, Y. Kobayashi, and M. Sato, J. Phys. Soc. Jpn. 79 (2010) 023702.

8 Y. Nakajima, T. Taen, Y. Tsuchiya, T. Tamegai, H. Kitamura, and T. Murakami, Phys. Rev. B 82, 220504 (2010).

9 H. Kontani and S. Onari, Phys. Rev. Lett. 104, 157001 (2010).

10 T. Saito, S. Onari, and H. Kontani, Phys. Rev. B 82, 144510 (2010).

11 S. Onari and H. Kontani, arXiv:1009.3882

12 C.-H. Lee, A. Iyo, H. Eisaki, H. Kito, M. T. FernandezDiaz, T. Ito, K. Kihou, H. Matsuhata, M. Braden, and K. Yamada, J. Phys. Soc. Jpn. 77, 083704 (2008).

13 T. Shimojima, F. Sakaguchi, K. Ishizaka, Y. Ishida, T. Kiss, M. Okawa, T. Togashi, C.-T. Chen, S. Watanabe, M. Arita, K. Shimada, H. Namatame, M. Taniguchi, K. Ohgushi, S. Kasahara, T. Terashima, T. Shibauchi, Y. Matsuda, A. Chainani, and S. Shin, Sccience 332, 564 (2011).

14 A. D. Christianson, E. A. Goremychkin, R. Osborn, S. Rosenkranz, M. D. Lumsden, C. D. Malliakas, I. S. Todorov, H. Claus, D. Y. Chung, M. G. Kanatzidis, R. I. Bewley, and T. Guidi, Nature 456, 930 (2008); Y. Qiu, W. Bao, Y. Zhao, C. Broholm, V. Stanev, Z. Tesanovic, Y. C. Gasparovic, S. Chang, J. Hu, B. Qian, M. Fang, and Z. Mao, Phys. Rev. Lett. 103, 067008 (2009); D. S. Inosov, J. T. Park, P. Bourges, D. L. Sun, Y. Sidis, A. Schneidewind, K. Hradil, D. Haug, C. T. Lin, B. Keimer, and V. Hinkov, Nature Physics 6, 178 (2010).

15 T. A. Maier and D. J. Scalapino, Phys. Rev. B 78, 020514(R) (2008); T. A. Maier, S. Graser, D.J. Scalapino, and P. Hirschfeld, Phys. Rev. B 79, 224510 (2009).

16 M. M. Korshunov and I. Eremin, Phys. Rev. B 78, 140509(R) (2008).

17 S. Onari, H. Kontani, and M. Sato, Phys. Rev. B 81, 060504(R) (2010)

18 M. Sato et al., private communications
19 T.A. Maier, S. Graser, P.J. Hirschfeld, and D.J. Scalapino, Phys. Rev. B 83, 220505(R) (2011).

20 S. Kasahara, T. Shibauchi, K. Hashimoto, K. Ikada, S. Tonegawa, R. Okazaki, H. Shishido, H. Ikeda, H. Takeya, K. Hirata, T. Terashima, and Y. Matsuda, Phys. Rev. B 81, 184519 (2010).

21 H. Kontani, Rep. Prog. Phys. 71, 026501 (2008); H. Kontani and K. Yamada, J. Phy. Soc. Jpn. 74, 155 (2005); H. Kontani, K. Kanki, and K. Ueda, Phys. Rev. B 59, 14723 (1999).

22 D. C. Jhonston, Advances in Physics 59, 803 (2010)

23 T. Shimojima, K. Ishizaka, Y. Ishida, N. Katayama, K. Ohgushi, T. Kiss, M. Okawa, T. Togashi, X.-Y. Wang, C.T. Chen, S. Watanabe, R. Kadota, T. Oguchi, A. Chainani, and S. Shin, Phys. Rev. Lett. 104, 057002 (2010).

24 Q. Wang, Z. Sun, E. Rotenberg, F. Ronning, E. D. Bauer, H. Lin, R. S. Markiewicz, M. Lindroos, B. Barbiellini, A. Bansil, D. S. Dessau, arXiv:1009.0271

${ }^{25}$ M. Yi, D. H. Lu, J.-H. Chu, J. G. Analytis, A. P. Sorini, A. F. Kemper, S.-K. Mo, R. G. Moore, M. Hashimoto, W. S. Lee, Z. Hussain, T. P. Devereaux, I. R. Fisher, Z.-X. Shen, PNAS 1086878.

26 T. Yildirim, Physica C 469 (2009) 425; F. Kruger, S. Kumar, J. Zaanen, and J. van den Brink, Phys. Rev. B 79, 054504 (2009); W. Lv, J. Wu, and P. Phillips, Phys. Rev. B 80, 224506 (2009); C.-C. Chen, B. Moritz, J. van den Brink, T. P. Devereaux, and R. R. P. Singh, Phys. Rev. B 80, 180418 (2009).

27 J.-H. Chu, J. G. Analytis, K. D. Greve, P. L. McMahon, Z. Islam, Y. Yamamoto, and I. R. Fisher, Science 329, 824 (2010); J. J. Ying, X. F. Wang, T. Wu, Z. J. Xiang, R. H. Liu, Y. J. Yan, A. F. Wang, M. Zhang, G. J. Ye, P. Cheng, J. P. Hu, and X. H. Chen, arXiv:1012.2731.

28 E. C. Blomberg, M. A. Tanatar, A. Kreyssig, N. Ni, A. Thaler, R. Hu, S. L. Bud'ko, P. C. Canfield, A. I. Goldman, and R. Prozorov, Phys. Rev. B 83, 134505 (2011).

29 A. Dusza, A. Lucarelli, F. Pfuner, J.-H. Chu, I.R. Fisher, L. Degiorgi, arXiv:1007.2543; M. Nakajima et al., unpublished.

30 R.M. Fernandes, L. H. VanBebber, S. Bhattacharya, P. Chandra, V. Keppens, D. Mandrus, M.A. McGuire, B.C. Sales, A.S. Sefat, and J. Schmalian, Phys. Rev. Lett. 105, 157003 (2010)

31 M. Yoshizawa, R. Kamiya, R. Onodera, Y. Nakanishi, K. Kihou, H. Eisaki, and C. H. Lee, arXiv:1008.1479.

32 M. Yoshizawa et al., private communication.

33 T. Goto, R. Kurihara, K. Araki, K. Mitsumoto, M. Akatsu, Y. Nemoto, S. Tatematsu, and M. Sato, J. Phys. Soc. Jpn. 80, 073702 (2011); T. Goto et al. (unpublised)

34 J. Zhao, D. T. Adroja, D.-X. Yao, R. Bewley, S. Li, X. F. Wang, G. Wu, X. H. Chen, J. Hu, and P. Dai, Nature Physics 5, 555 (2009). 
35 W.A. Little, Phys. Rev. 134, A1416 (1964)

36 V.L. Ginzburg, Zh. Eksp. Teor. Fiz. 472318 (1964) [Sov. Phys. JETP 20, 1549 (1965)]

37 J. E. Hirsch and D. J. Scalapino, Phys. Rev. Lett. 56, 2732 (1986)

38 V. Cvetkovic and Z. Tesanovic, EPL, 85 (2009) 37002

39 M. Berciu, I. Elfimov, and G. A. Sawatzky, arXiv:0811.0214

40 T. Takimoto, T. Hotta, T. Maehira and K. Ueda, J. Phys.: Condens. Matter 14, L369 (2002).

41 Y. Yanagi, Y. Yamakawa, and Y. Ono, Phys. Rev. B 81, $054518(2010)$

42 T. Miyake, K. Nakamura, R. Arita, M. Imada, J. Phys. Soc. Jpn. 79, 044705 (2010)

43 Y. Yanagi, Y. Yamakawa, N. Adachi, and Y. Ono, J. Phys. Soc. Jpn. 79 (2010) 123707

44 H. Kontani and S. Onari, J. Phys. Soc. Jpn., 80, 056001 (2011).

45 J.R. Schrieffer, theory of superconductivity (Benjamin, New York, 1964)

46 In Ref. 43, $\chi_{x^{2}-y^{2}}^{Q}(\boldsymbol{q}, 0)$ due to the "othrorhombic phonon" (= $g_{66}$ in the present paper) is incommensurate with momentum $\boldsymbol{q} \approx(0.35 \pi, 0)$. In the present tight-binding model $^{2}, \chi_{x^{2}-y^{2}}^{Q}(\boldsymbol{q}, 0)$ due to $g_{66}$ has the highest peak at $\boldsymbol{q} \approx(\pi, 0)$ due to the nesting between electron- and holepockets, while it is hardly enhanced at $\boldsymbol{q}=\mathbf{0}$.

47 K. Yada and H. Kontani, Phys. Rev. B 77, 184521 (2008); K. Yada and H. Kontani, J. Phys. Soc. Jpn. 75, 033705 (2006)

48 In Ref. 10, we claimed that the enhancement of $\chi_{x z}^{Q}(\mathbf{0}, 0)$ in the five-orbital model induces the softening of $C_{44}$. However, it was incorrect since the effect of unfold-gauge transformation on $\chi_{x z}^{Q}(\mathbf{0}, 0)$ was overlooked. In fact, $\chi_{x z}^{Q}(\mathbf{0}, 0)$ is not enhanced in the ten-orbital model.

49 H. Kontani, and K. Yamada, J. Phys. Soc. Jpn. 65, 172
(1996)

50 L. Boeri, O. V. Dolgov, and A. A. Golubov, Phys. Rev. Lett. 101, 026403 (2008)

51 P. Thalmeier and B. Luthi, in Handbook on the Physics and Chemistry of Rare Earths Vol. 14, ed K A Gschneidner Jr and L Eyring (Amsterdam: Elsevier) p 245 (1991).

52 P. M. Levy, P. Morin and D. Schmitt, Phys. Rev. Lett. 42, 1417 (1979)

53 L. G. Aslamasov and A. I. Larkin, Soviet Phys.-SolidState 10, 875 (1968).

54 H. Fukuyama, H. Ebisawa, and T. Tsuzuki, Prog. Theor. Phys. 46 (1971) 1028.

55 I. Ussishkin, Phys. Rev. B 68, 024517 (2003)

56 T. Moriya, Spin Fluctuations in Itinerant Electron Magnetism (Springer-Verlag, 1985); T. Moriya and K. Ueda, Adv. Physics 49555 (2000); T. Moriya and K. Ueda : Rep. Prog. Phys. 66 (2003) 1299.

57 A.A. Abricosov, L.P. Gor'kov and I.E. Dzyaloshinskii, Methods of Quantum Field Theory in Statistical Physics (Dover, New York, 1975).

${ }^{58}$ H. Shishido, A. F. Bangura, A. I. Coldea, S. Tonegawa, K. Hashimoto, S. Kasahara, P. M. C. Rourke, H. Ikeda, T. Terashima, R. Settai, Y. Onuki, D. Vignolles, C. Proust, B. Vignolle, A. McCollam, Y.Matsuda, T. Shibauchi, A. Carrington, Phys. Rev. Lett. 104, 057008 (2010).

59 K. Kubo and P. Thalmeier, J. Phys. Soc. Jpn. 78, 083704 (2009); C.-C. Lee, W.-G. Yin, and W. Ku, Phys. Rev. Lett. 103, 267001 (2009); H. Oh, D. Shin, and H. J. Choi, arXiv:1012.2224.

60 K. Sugimoto, E. Kaneshita, and T. Tohyama, J. Phys. Soc. Jpn. 80 (2011) 033706.

61 H. Kontani, unpublished.

62 C.-C. Chen, J. Maciejko, A. P. Sorini, B. Moritz, R. R. P. Singh, and T. P. Devereaux, Phys. Rev. B 82, 100504(R) (2010). 